\title{
Stage-specific, morphological and molecular markers of encystation in Giardia lamblia
}

1 Elizabeth B. Thomas ${ }^{1}$, Renaldo Sutanto ${ }^{1,2}$, Richard S. Johnson ${ }^{3}$, Han-Wei Shih ${ }^{1}$, Jana

2 Krtková $^{4}$, Michael J. MacCoss ${ }^{3}$, Alexander R. Paredez ${ }^{*}$

$3{ }^{1}$ Department of Biology, University of Washington, Seattle, Washington, United States

$4{ }^{2}$ Department of Bioengineering and Therapeutic Sciences, University of California San Francisco,

5 San Francisco, California, United States of America

$6 \quad{ }^{3}$ Department of Genome Sciences, University of Washington, Seattle, Washington, United States

$7 \quad{ }^{4}$ Department of Experimental Plant Biology, Faculty of Science, Charles University, Prague, Czech

8 Republic

* Correspondence:

Corresponding Author

12 aparedez@uw.edu

13 Keywords: Giardia1, Encystation2, Rho GTPase, Rac4, Membrane trafficking, ESV 6 (Min.514 Max. 8)

15 Word length 4997 words

16 No. of Figures and Tables Four figures and one Table; Five Supplementary Figures and three

17 Supplementary Tables

18 Abstract

19 Differentiation into environmentally resistant cysts is required for transmission of the ubiquitous 20 intestinal parasite Giardia lamblia. Encystation in Giardia requires the production, processing and transport of Cyst Wall Proteins (CWPs) in developmentally-induced, Golgi-like, Encystation Specific Vesicles (ESVs). Progress through this trafficking pathway can be followed by tracking CWP localization over time. However, there is no recognized system to distinguish the advancing stages of this process which can complete at variable rates depending how encystation is induced. Here we propose a staging system for encysting Giardia based on the morphology of CWP1-stained ESVs. We demonstrate the molecular distinctiveness of maturing ESVs at these stages by following GlRab GTPases through encystation. Previously, we established that Giardia's sole Rho family GTPase, $G l R a c$, associates with ESVs and has a role in regulating their maturation and the secretion of their cargo. As a proof of principle, we delineate the relationship between GlRac and ESV stages. Through proteomic studies, we identify putative interactors of $G l$ Rac that could be used as additional stagespecific ESV markers. This staging system provides a common descriptor of ESV maturation regardless of the source of encysting cells. Furthermore, the identified set of molecular markers for ESV stages will be a powerful tool for characterizing trafficking mutants that impair ESV maturation and morphology. 


\section{Importance}

Giardiasis is a diarrheal disease that affects 280 million people worldwide. It is caused by Giardia lamblia, a protozoan parasite which rely on differentiating from host-dwelling trophozoites to environmentally-resistant cysts for transmission and survival. This encystation process requires the transport of Cyst Wall Proteins (1-3) within membrane-bound compartments called Encystation Specific Vesicles (ESV) from the endoplasmic reticulum to the surface of the cell. The whole process takes 24 hours to complete and these compartments are the only recognizable equivalent of Golgi apparatus in this minimalistic organism. Progress of this trafficking pathway can be followed by localizing Cyst Wall Protein 1 over time post induction of encystation but this can be ambiguous when specific molecular events need to be specified. Here we propose a staging system that is based on ESV morphology changes by capitalizing on the secretory/processing events we already know they represent. We validate the molecular distinctiveness of these stages by following Giardia Rabs through the pathway and characterize putative interactors of an established regulator of encystation, GlRac, to provide additional stage-specific molecular markers. This staging system will provide a definitive, yet adaptable, framework to map out functions of yet-to-be discovered players of this important pathway.

\section{Introduction}

Giardia lamblia (syn. Giardia intestinalis and Giardia duodenalis) is a major intestinal parasite which infects more than 280 million people every year (Lane \& Lloyd, 2002). The lifecycle of this diplomonad protozoan is simple, featuring only two stages - the binucleate, double-diploid, proliferative trophozoites which non-invasively colonizes host intestines and the environmentallyresistant, infectious, non-motile cysts that are shed in host's feces. Regulation of encystation ensures the production of viable cysts and promotes transmission of this ubiquitous parasite. Being a popular life-cycle strategy also adopted by other protozoan parasites, studying this differentiation process is important and Giardia is the best-developed model available (Eichinger, 2001).

Giardia encystation requires the construction of its protective cyst wall, an extracellular matrix composed of Cyst Wall Material (CWM). CWM contains three paralogous Cyst Wall Proteins (CWP1-3) and a unique $\beta$-1,3-linked N-acetylgalactosamine (GalNAc) homopolymer (Gerwig et al., 2002; Lujan et al., 1995; Sun et al., 2003). When induced, large quantities of CWPs are synthesized and transported from the endoplasmic reticulum (ER) to the cell surface in membrane-bound organelles called Encystation-Specific Vesicles (ESVs). Giardia lacks classical Golgi apparatus. However, since nascent ESVs arise from ER-exit sites (ERES; Faso et al. 2013) and are marked by several Golgi markers they are thought to be developmentally-induced Golgi (Marti et al., 2003). This view is supported by the roles ESVs play as the only recognizable post-ER delay compartments. ESVs feature machinery needed for the post-translational processing and subsequent partitioning of CWPs into distinct phases (Davids et al., 2004; Reiner et al., 2001; Slavin et al., 2002). After proteolytic cleavage of CWP2, CWP1 and the N-terminal end of cleaved CWP2 are sorted into the outer fluid phase while CWP3 and C-terminal end of cleaved CWP2 remain as the inner condensed core (DuBois et al., 2008; Konrad et al., 2010; María C. Touz et al., 2002). Additionally, ESVs coordinate secretion of CWM to the cell surface, likely mediated by a higher order networking structure (Štefanić et al., 2009). Processed CWPs are deposited sequentially; the fluid phase first at a rapid rate where binding of CWP1 to the cell surface is mediated by its lectin binding domain that recognizes GalNac fibrils on the surface of the encysting cell (Chatterjee et al., 2010). This is followed by slower secretion of the condensed phase (Konrad et al., 2010; Štefanić et al., 2009). These events are trackable by following ESV morphology and CWP localization. 
As a lab-inducible and -tractable secretory pathway of a minimalistic organism, Giardia's encystation process provides a unique opportunity to uncover the constraining principles of membrane trafficking. Despite fundamental differences in compartment organization, canonical membrane trafficking players continue to perform conserved roles in Giardia (Maria C. Touz \& Zamponi, 2017). The accumulation of CWP and de novo ESV biogenesis at the ERES is dependent on COPII and the small GTPase, SarI - vesicle coat proteins that transport cargo from the rough ER to the Golgi apparatus in higher eukaryotes (Štefanić et al., 2009). Additionally, Arf1, a small GTPase that canonically plays a central role in intra-Golgi transport by regulating COPI and clathrin membrane coats, is required for ESV maturation; inhibiting Arf1 activity interfered with the transport and secretion of CWM to the cell surface (Štefanić et al., 2009). The sole Rho GTPase in Giardia, $G l$ Rac, whose homologs are known to coordinate vesicle trafficking and the cytoskeleton in plants and animals, was found to regulate CWP trafficking and secretion (Krtková et al., 2016).

Similar to its homologs, GlRac is thought to regulate Giardia encystation by acting as a molecular switch for the recruitment and regulation of effector proteins which drive the progress of encystation. We previously demonstrated that $G l \mathrm{Rac}$ is required for the temporal coordination of CWP1 production, ESV maturation, and CWP1 secretion (Krtková et al., 2016). The relationship between GlRac and its roles in CWP trafficking are complicated by the dynamic association of GlRac with ESVs (Krtková et al., 2016). While it is recognized that ESVs go through stages of maturation (Konrad et al., 2010), there is no established criteria for specifically identifying these stages. Additionally, timing post induction of encystation has been used to stage ESVs, but the amount of time it takes to encyst varies by the method used to promote encystation (Einarsson et al., 2016; Konrad et al., 2010; Luján et al., 1996). For a direct comparison of CWP1 and CWP2 induction using these methods see Fig. S1. Our efforts to specify the molecular events that coincided with GlRac activity at ESVs, highlighted the need for a standardized system to distinguish the advancing stages of encystation. Here, we develop a system for staging encysting Giardia cells that is based on our current understanding of molecular events during encystation and observed by changes in ESV morphology (Konrad et al., 2010). Furthermore, this would allow us to navigate around ambiguities introduced by variation in the timing and efficiency of encystation when different protocols for induction are used. Our survey of Giardia Rabs, small GTPases that are known to control the specificity and directionality of membrane trafficking pathways and mark specific organelles, highlight the molecular distinctiveness between the stages we propose. As a proof of principle, we characterized putative interactors of $G I \mathrm{Rac}$ as identified by proteomics and confirm their association with ESVs. This work would facilitate future studies where the functions of GlRac effectors would

114 be precisely mapped in this crucial trafficking pathway.

\section{Materials and Methods}

\section{Strain and culture conditions.}

117 G. lamblia strain WB clone C6 (ATCC 50803; American Type Culture Collection) was cultured in

118 TYDK medium (per 100ml; $2 \mathrm{~g}$ casein digest, $1 \mathrm{~g}$ yeast extract, $1 \mathrm{~g}$ glucose, $0.2 \mathrm{~g} \mathrm{NaCl}, 0.06 \mathrm{~g}$ $119 \mathrm{KH}_{2} \mathrm{PO}_{4}, 0.095 \mathrm{~g} \mathrm{~K}_{2} \mathrm{HPO}_{4}, 0.2 \mathrm{~g}$ L-cysteine, $0.02 \mathrm{~g}$ L-ascorbic acid, $1.2 \mathrm{mg}$ ferric ammonium citrate) supplemented with $10 \%$ adult bovine serum with $\mathrm{pH}$ adjusted to 7.1. To induce encystation in trophozoites, the two-step encystation protocol was followed. Trophozoite cultures were first grown to confluency $\left(\sim 1 \times 10^{6}\right.$ cells $\left./ \mathrm{ml}\right)$ for $36 \mathrm{~h}$ in pre-encystation medium (same as growth medium above but without bile and $\mathrm{pH}$ at 6.8). Encystation was induced by switching culture medium to encystation medium (same as growth medium but $\mathrm{pH} 7.8$ and supplemented with $10 \mathrm{~g} / \mathrm{l}$ bovine/ovine bile instead of bovine bile) and incubating the cells further for either $8 \mathrm{~h}$ or 24 has indicated. 


\section{Vector construction}

127 All constructs were made using traditional cloning, Gibson assembly (Gibson et al., 2009) or In-

128 Fusion kit (Takara Bio); for full details and Gene Accession numbers, consult Table S1. Note,

129 constructs made to tag Giardia Rabs and putative GlRac-interactors were designed for stable

130 integration by homologous recombination into the Giardia genome for endogenous expression.

131 Generation of the native promoter morpholino-sensitive (ms) HALO_C18_GlRac_PuroR (designed

132 for endogenous expression) and PAK promoter_CRIB_N11_mNG_3HA_NeoR (designed for

133 episomal expression) constructs have been described previously (Hardin et al., 2021, submitted).

134 Plasmid backbones used to build constructs in this paper were sourced from Gourguechon \& Cande,

135 2011, Krtková et al., 2016, Michaels et al., 2020 and Paredez et al., 2014.

136 For transfection, 5 to $50 \mu \mathrm{g}$ of DNA was electroporated $(375 \mathrm{~V}, 1,000 \mu \mathrm{F}, 750 \Omega$; GenePulser Xcell; 137 BioRad, Hercules, CA) into trophozoites. Following electroporation, the cells were added to $13 \mathrm{ml}$ pre-warmed, fresh TYDK medium and allowed to recover at $37^{\circ} \mathrm{C}$ overnight before beginning selection with G418 or Puromycin for 4-7 days. Strains were maintained at a final concentration of $14030 \mu \mathrm{g} / \mathrm{ml} \mathrm{G} 418$ or $0.3 \mathrm{mg} / \mathrm{ml}$ Neomycin.

\section{Luciferase assay}

142 Confluent cell cultures were incubated with the three different encystation media (two-step, Uppsala, 143 and lipoprotein-deficient) for $8 \mathrm{~h}$ and $20 \mathrm{~h}$, then pelleted and resuspended with Hepes-Buffered 144 Saline. $200 \mu$ l diluted cells $\left(2 \times 10^{4}\right.$ cells $)$ and $50 \mu 1(8 \mathrm{mg} / \mathrm{ml})$ D-luciferin (GoldBio, USA) were 145 loaded into each well. Plates were incubated for time increment from $5 \mathrm{~min}$ to $30 \mathrm{~min}$ at $37^{\circ} \mathrm{C}$. 146 Luciferase activity was determined with an Envision MultiLabel Plate Reader (Perkin Elmer, USA).

\section{Immunofluorescence microscopy.}

148 Immunofluorescence assays were performed as described previously (Krtková et al., 2016), To detect 3HA tag, anti-HA rat monoclonal antibodies 3F10 (Roche) diluted to 1:125 followed by Alexa 488-

150 conjugated anti-rat antibody (Molecular probes) diluted to 1:250 were used. To detect HALO tag $1510.5 \mu \mathrm{M}$ Janelia Fluor 549 (Promega) dye or HaloTag® TMR Ligand (Promega) were used. CWP1 152 was detected with Alexa 647-conjugated anti-CWP1 antibody (Waterborne, New Orleans, LA)

153 Fluorescent images were acquired on DeltaVision Elite microscope using a 100×, 1.4-numerical aperture objective and a PCO Edge sCMOS camera. Deconvolution was performed with SoftWorx (API, Issaquah, WA) and images were analyzed using Fiji, ImageJ (Schindelin et al., 2012). Pearson Coeffecient analysis and Costes' automatic thresholding were obtained using the JACoP plugin for ImageJ (Bolte \& Cordelieres, 2006). 3D viewing and manual scoring of cells were performed using Imaris (Bitplane, version 8.9) Figures were assembled using Adobe Photoshop.

\section{Affinity purification}

160 Affinity purification of OneSTrEP-GlRac was done according to a previously used protocol (Paredez 161 et al., 2014) Note that two-step encystation protocol was followed during culturing for the $8 \mathrm{~h}$ p.i.e. 162 experiments. The resulting elutes were analyzed with Liquid Chromatography with tandem mass

163 spectrometry. 


\section{Mass Spectrometry}

166 Samples were prepared using the FASP method (Wiśniewski et al., 2009). Briefly, the samples were concentrated in an Amicon Ultra 10K filter, washed twice with $25 \mathrm{mM}$ ammonium bicarbonate (ABC), and the disulfides reduced using $10 \mathrm{mM}$ tris 2-carboxyethyl phosphine (TCEP) for $1 \mathrm{~h}$ at $37^{\circ} \mathrm{C}$. The resulting thiols were alkylated with $12 \mathrm{mM}$ iodoacetamide for 20 min prior to spinning out the liquid. The proteins were washed twice with $100 \mu 150 \mathrm{mM} \mathrm{ABC}$. Digestion occurred after addition of $1 \mu \mathrm{g}$ trypsin (Promega sequencing grade) in $200 \mu 150 \mathrm{mM} \mathrm{ABC}$, and overnight incubation at $37^{\circ} \mathrm{C}$. The peptides were spun out of the filter and dried using a vacuum centrifuge.

All mass spectrometry was performed on a Velos Pro (Thermo) with an EasyLC HPLC and autosampler (Thermo). The dried pulldowns were solubilized in $25 \mu 1$ of loading buffer $(0.1 \%$ trifluoroacetic acid and $2 \%$ acetonitrile in water), and $6 \mu \mathrm{l}$ was injected via the autosampler onto a $150-\mu \mathrm{m}$ Kasil fritted trap packed with Reprosil-Pur C18-AQ (3- $\mu \mathrm{m}$ bead diameter, Dr. Maisch) to a bed length of $2 \mathrm{~cm}$ at a flow rate of $2 \mu \mathrm{l} / \mathrm{min}$. After loading and desalting using a total volume of $8 \mu \mathrm{l}$ of loading buffer, the trap was brought on-line with a pulled fused-silica capillary tip (75- $\mu \mathrm{m}$ i.d.) packed to a length of $25 \mathrm{~cm}$ with the same Dr. Maisch beads. Peptides were eluted off the column using a gradient of $2-35 \%$ acetonitrile in $0.1 \%$ formic acid over $120 \mathrm{~min}$, followed by $35-60 \%$ acetonitrile over $5 \mathrm{~min}$ at a flow rate of $250 \mathrm{nl} / \mathrm{min}$. The mass spectrometer was operated using electrospray ionization $(2 \mathrm{kV})$ with the heated transfer tube at $25^{\circ} \mathrm{C}$ using data dependent acquisition (DDA), whereby a mass spectrum ( $\mathrm{m} / \mathrm{z} 400-1600$, normal scan rate) was acquired with up to 15 MS/MS spectra (rapid scan rate) of the most intense precursors found in the MS1 scan.

Tandem mass spectra were searched against the protein sequence database that was downloaded from GiardiaDB, using the computer program Comet (Eng et al., 2013) Iodoacetamide was a fixed modification of cysteine, and oxidized methionine was treated as a variable modification. Precursor mass tolerance was $2 \mathrm{Da}$, and fragment ion tolerance was +/- 0.5 Da. Discrimination of correct and decoy spectra was performed using Percolator (Käll et al., 2007) with a 1\% q-value cutoff. Proteins that had more than one unique peptide and significantly higher values for normalized total spectral counts (as determined by Fisher Exact Test with a Bonferroni multiple testing correction with an alpha of 0.01) in the OneSTrEP-GlRac pulldown sample compared to its counterpart WT control sample, were noted to be hits.

\section{Results}

\section{Encystation Staging}

196 Encystation was induced with a two-step protocol where Giardia trophozoites were initially cultured 197 in no-bile, low-pH media ( $\mathrm{pH}$ 6.8) and then moved into high-bile, high $\mathrm{pH}$ media ( $\mathrm{pH} 7.8$ ) (Boucher 2010). In our hands, while some level of synchronization could be achieved from this method, cells remained in various stages within the process of cyst development, as judged by localizing CWP1 and visualizing ESV morphology (Fig. 1). We, therefore, sought to increase the resolution of staging encysting cells by going beyond noting hours post induction of encystation (h p.i.e.) and categorizing each cell based on the morphology of CWP1-stained ESVs instead (Fig. 1).

Based on our current understanding of the sequence of events involved in encystation, we propose the following key for staging encysting cells (Fig. 1): Stage I -CWP1 localizes to the ER; Stage II CWP1 localize in the ER and also in ER-associated punctate structures thought to be ER-exit sites (Faso et al., 2013); Stage III - CWP1 localizes homogenously in small ESVs; Stage IV - CWP1 
localize to doughnut-shaped structures as a result of CWP2 being proteolytically processed to drive core condensation which pushes fluid phase CWP1 to the vesicle periphery. Stage V - found in cultures induced to encyst longer than $8 \mathrm{~h}$ (24 h p.i.e in this study); CWP1 now localizes to large doughnut-shaped structures of matured ESVs about to be partitioned from condensed-phase cyst wall material (CWM) into separate vesicles. Stage VI - CWP1 is present in smaller separate vesicles that are close to the surface of the cells and ready to be secreted out to form the cyst wall. Our focus here is on ESV maturation therefore we did not analyze partially to fully formed cysts. Nonetheless, these staging parameters can be expanded to include them in the future.

\section{Giardia Rab GTPases associate to ESVs in stage-specific manner}

217 It is well established that different compartments of the membrane trafficking pathway feature unique molecular identities. Rab GTPases, through the recruitment of specific effectors, regulate the trafficking of each other through the endocytic/sorting/secretory pathway (Pfeffer, 2017). The resulting spatio-temporal specific recruitment of Rabs help direct cargo traffic. Rab GTPases can, therefore, be used as identity markers for differentiating trafficking compartments. We hypothesized that a subset of the nine Rab GTPases encoded in the Giardia genome would also demarcate ESVs as they mature, providing unique molecular markers for different stages of these compartments as they sort and secrete CWPs. Each GIRab was tagged on the N-terminus with mNeonGreen (mNG) to visualize their localization and the cells were subjected to the two-step encystation process before being processed for immunofluorescence assays. Cells were staged as described above and the degree of colocalization between CWP1 and the GlRab GTPases were determined manually.

To cross-check our qualitative scoring, colocalization was verified by calculating Pearson statistical measurements are not effective for highlighting qualitative differences when proteins display complex localization patterns (Bolte \& Cordelières, 2006; Fig. S2). Therefore, we manually scored 15 to 20 cells per stage; after viewing images in 3D, they were given a score between $0-5$, with higher scores being granted to cells featuring more robust recruitment of tagged-Rab proteins colocalizing with CWP1-stained vesicles (Fig. S3). Consistency between scores assigned by two team members for a sample subset of cells gave us confidence that this approach is reproducible. Of interest, were Rabs with CWP1 colocalization scores that peaked at the different encystation stages GlRab2a at Stages I and II, GlRabA at Stage III, GlRab D at Stage IV and GlRab1a at Stages V and VI (Fig. 2). GlRab32 did not localize to ESVs at any of the stages we looked at (Fig. S3). Multiple attempts at tagging GlRab2b were unsuccessful and therefore this Rab GTPase was not included in our analysis. Our data is consistent with the cisternal maturation model where ESVs cargo remains in place and the molecular identity of the compartment changes as CWP is processed and sorted (Mani \& Thattai, 2016). Here, we have shown that the staging of ESVs via their morphologies demarcates ESVs undergoing unique molecular events and provides a better framework for dissecting ESV molecular biology and the developmental state of encysting cells.

\section{GlRac activity during encystation is stage-specific}

Small Rho GTPase proteins, by cycling between an active GTP-loaded conformation and an inactive GDP-bound conformation, act as molecular switches that spatially and temporally regulate the recruitment of effector proteins. We previously noted that Giardia's sole Rho GTPase, GlRac ${ }_{2}$ has variable association with ESVs (Krtková et al., 2016), but we did not determine the encystation stage-specificity of it. GlRac was endogenously tagged to express HALO on its N-terminus and the cells were subjected to the same ESV colocalization assay as described above. HALO-GlRac strongly colocalizes with CWP1 during Stage I, an association which wanes through the mid-stages, 
and then increases at Stages V and VI of encystation (Fig. 3A). This is consistent with the proposed activities of $G l$ Rac during encystation. A large proportion of $G l$ Rac is thought to be sequestered in its inactive form at the ER while GlRac association with mid-stage and late-stage ESVs promotes ESV maturation and CWP secretion, respectively (Krtková et al., 2016). We then used a CRIB domainbased Rho GTPase biosensor (Manser et al., 1994; Srinivasan et al., 2003), CRIB-mNG, to confirm our functional analyses. CRIB-mNG, which marks active GTP-loaded GlRac, colocalized with ESVs as their CWP cargo were being first sorted into condensed and fluid phases i.e. Stages III and IV, and also at Stage VI of encystation when ESVs have completed undergoing the second sorting step and were beginning to secrete their cargo to form the cyst (Fig. 3B). This pattern of localization by CRIB-mNG indicates that the ER associated population of GlRac is GDP-loaded while the GlRac associated with ESVs is GTP-loaded.

\section{Putative effectors of $G l$ Rac during Giardia encystation}

Next, we sought to identify the effectors regulated by GlRac during encystation - in this study we specifically focused on mid-stage encysting cells featuring ESVs that associate with active GlRac. Giardia cells exogenously expressing N-terminally OneSTrEP (OS-) tagged GlRac were induced to encyst using the two-step method. We then performed an affinity purification with OS-GlRac lysates at $8 \mathrm{~h}$ p.i.e. Purifications were completed in triplicate, and wild type (WT) trophozoites were used as a control to identify non-specific binding to the Strep-Tactin beads. The resin eluates were then analyzed via mass spectrometry to identify putative interactors of $G l$ Rac. We identified 57 proteins, statistically significantly associating with $G l$ Rac in at least one replicate, in both trophozoites and encysting cells. Similarly, 33 proteins associated with $G l$ Rac exclusively in non-encysting trophozoites while 28 proteins were associated with encysting cells. The complete list, including lowabundance hits and proteins also identified in our mock control, is given in Table S3. Altogether, we identified 18 proteins predicted to have a role in membrane trafficking that were enriched in our encysting population (Table S4). Out of these, four were previously known to associate with ESVs supporting the idea that our list could have additional ESV components. We focused on 11 proteins that were homologs of known players of membrane trafficking in other eukaryotes (Table 1). GlRab1a and GlRab2a had already been visualized earlier (Fig. S3). Each of the rest of the candidate genes were endogenously expressed with a dual tag of triple hemagglutinin (HA) fused to $\mathrm{mNG}$ in cells already endogenously expressing Halo-GlRac. The candidates were then visualized via immunofluorescence assays along with GlRac and CWP1 (Fig. S4). Six of the eight candidates listed showed patterns of colocalization with CWP1 similar to that of GlRac - GlRab2a (Fig. 2), GlSec61$\alpha$, GlCoatomer $\alpha$ subunit, GlCoatomer $\beta$ ' subunit and Glv-SNARE (Fig. 4) suggesting that they might be involved in the same pathway regulating encystation as GlRac.

\section{Discussion}

288 Here, we have developed a staging system for encysting cells and have identified novel molecular markers for the specific ESV stages that correlate with the progression of encystation. We previously noted that GlRac associates with ESVs at defined points of their maturation process (Krtková et al., 2016). The lack of an established method to unambiguously pinpoint progression of encystation in individual cells, along with the variability of encystation rates that result from different in vitro encystation protocols, prompted us to develop a staging system that is based on ESV morphologies and independent of the method used to induce encystation. The encystation stage-specific recruitment of these molecular markers is consistent with the cisternal maturation model (Mani \& Thattai, 2016) and the observations of Štefanić et al., 2009. Briefly, ESVs are thought to act as developmentallyinduced Golgi (Marti et al., 2003). CWPs are first detected approximately two hours after the 
298

299

300

301

302

303

304

305

306

307

308

309

310

311

312

313

314

315

316

317

318

319

320

321

322

323

324

325

326

327

328

329

330

331

332

333

334

335

336

337

338

339

340

341

342

343 induction of encystation (two-step protocol); the export of CWPs from the ER, maturation of ESVs and secretion of processed CWPs to the plasma membrane together represent a simplified version of the Golgi cisternal maturation model. CWP export from the ER was found to require functional Arf1, Sar1 and Rab1 (Štefanić et al., 2009). ESVs generated de novo from this export process at ERESs contain pre-sorted material that is simultaneously transported and processed.

Staging ESV maturity previously relied on timing p.i.e. or simply referring to encysting cells as "early" or "late" without a standardized criteria for making these distinctions (Einarsson et al., 2016; Konrad et al., 2010; Luján et al., 1996). Timing p.i.e. is a practical approach to experimentation and can even be meaningful when comparing cells exposed to the same encystation medium when broad trends are being studied. Yet, this method is imprecise due to in vitro encystation protocols being only semi-synchronous. It is thought that there is a restriction point that prevents cells outside of G2 from entering the encystation response (Reiner et al., 2008). Given an eight-hour cell cycle it is not surprising that we find cells at differing encystation stages when examining induced populations. Additionally, the timing and efficiency of inducing encystation varies between the three main in vitro encystation methods - cholesterol starvation (Luján et al., 1996), the Uppsala method (Einarsson et al., 2016), and the two-step encystation protocol (used in this study). To a smaller extent lot-to-lot variation of serum and bile components of growth medium and encystation medium impact doubling times and, therefore, the efficiency of inducing encystation. Most importantly, in vivo studies employing animal models of infection currently cannot be synchronized and timing h p.i.e would not be informative. Recent efforts to study the encystation response in vivo has raised questions about whether in vitro studies can recapitulate in vivo encystation dynamics (Pham et al., 2017). Thus, the emerging view is that in vitro observations should be verified in vivo and this encystation staging system would also be a practical way to characterize the distribution of encysting cells within host intestines.

Our universally applicable staging system will allow the field to standardize encystation staging regardless of the method of induction used. Each ESV morphology change is thought to correspond with sequential molecular events, including secretion from the ER, CWP processing, sorting, and pulsed cellular secretion of processed CWP. ESV morphology changes therefore provide landmarks for encystation stages. Observing and categorizing ESV morphologies are obvious and easy to follow, therefore making it accessible for adoption by the wider Giardia research community. This would be advantageous since it would reduce ambiguity when the timeline of molecular pathways directing CWP traffic are being specified. Finally, as the resolution of these events increase, encystation stages could be further subdivided to accommodate new discoveries.

Beyond morphological categorization we have identified several Giardia Rab GTPases that demarcate specific encystation stages, indicating that our staging system corresponds to unique molecular identities. Some Giardia Rab GTPases were previously known to be associated with ESVs and overall this group of proteins is upregulated during encystation (Einarsson et al., 2016; Marti et al., 2003). Here we tagged and followed eight out of nine Giardia Rab GTPases over the course of encystation. Seven of the eight Rab GTPases analyzed here associated with ESVs. By scoring the degree of colocalization with CWP1 during the different encystation stages, we were able to identify their distinct patterns of ESV association thus potentially indicating the point at which their functions were required as encystation progressed. Functional equivalence between some of the GlRabs and their homologs can be inferred. GlRab2a and CWP1 colocalization peaked at Stages I and II, paralleling its mammalian homologs which reside in the ER-to-Golgi Intermediate Compartments (ERGIC) and regulate Golgi biogenesis, and bidirectional transport between the two compartments (Saraste, 2016). GlRabD shows the closest homology to Rab8 or Rab13 known to localize at the 
344 trans Golgi network, recycling endosomes, late endosomes and the plasma membrane. They have

345 especially been implicated in biosynthetic and recycling endosomal pathways (Ioannou \&

346 McPherson, 2016). In G. lamblia, RabD colocalization with ESVs peaks at Stage IV and equivalence

347 could be argued.

348 While the similarities between Giardia Rabs and their eukaryotic homologs allow for some inferred

349 function, not all Giardia Rabs have clear orthologs. The closest homologs of GlRabA (RabA in

350 plants/Rab 4 or Rab11 in mammals) are known to associate with the trans Golgi and post-Golgi

351 networks, specifically, the recycling endosomes (Li \& Marlin, 2015; Minamino \& Ueda, 2019;

352 Vernoud et al., 2003) and GlRabA was shown to associate with ESVs the most at Stage III, which is

353 likely equivalent to cis Golgi earlier in canonical secretory pathway. Additionally, GlRab1a

354

355

356 colocalization with ESVs peaks at Stages V and VI which is when the ESVs undergo further sorting and the fluid phase of ESVs are beginning to disassociate for secretion. This is different from canonical Rab1 isoforms that have been established to regulate transport between ER-ERGIC-Golgi interface (Saraste, 2016).

358 The importance of Rabs in regulating Giardia encystation is yet to be fully understood. Previously, GlRabla was shown to be necessary for ESV development and cyst wall formation (Štefanić et al., 2009) complementing the observations we have made here. The specific functions of GlRabs would make an interesting topic for future studies.

As a proof of principle for our staging system, we turned to $G l$ Rac which has a complex relationship to ESVs. In agreement with previously published data, GlRac colocalization with CWP1 peaked at stages I and VI with low level colocalization being detectable throughout the rest of the stages. GlRac was hypothesized to be sequestered in the ER in an inactive state and then have a role in promoting ESV maturation and secretion of CWP1 (Krtková et al., 2016). Based on our hypothesis, GlRac was expected to be active at Stages III/IV and VI which we confirmed using CRIB-mNG as a GlRac signaling biosensor. As a molecular switch, $G l$ Rac is expected to recruit effectors that promote maturation of ESVs and secretion of CWP1.

To identify GlRac effector proteins we affinity purified $G l$ Rac interactors from non-encysting and encysting populations. We were not surprised to find many statistically significant interacting proteins since GlRac, as the sole Rho GTPase in Giardia, is presumably responsible many of the same roles the multitude of Rho GTPases carry out in other eukaryotes (Hodge \& Ridley, 2016; Lawson \& Ridley, 2018; Phuyal \& Farhan, 2019). Here we chose to focus on proteins predicted to have a role in trafficking as we had the potential to identify novel ESV components. Indeed, every protein selected from the proteomics hits associated with the ER and/or ESVs with GlRac.

A number of candidates displayed patterns of colocalization that were similar to the patterns displayed by $G l$ Rac with CWP1, including $G l$ Sec61- $\alpha$, GlCoatomer- $\alpha$ subunit, $G l$ Coatomer- $\beta$ ' subunit and Glv-SNARE. Each of these proteins was found to be in the ER at Stage I with high colocalization scores with CWP1 which reduced in subsequent stages to rise back up again in the final two stages. This would suggest that they localize in the same compartments during encystation. Effector proteins can also bind the inactivated GDP-bound form of Rho GTPases, so it is not surprising that some of these candidates colocalize with $G l \mathrm{Rac}$ at the ER where $G l \mathrm{Rac}$ is largely GDP-loaded. All of these except $G l v-S N A R E$ were also pulled down by $G l$ Rac in trophozoites indicating additional constitutive roles, while $G l \mathrm{NSF}, G l \alpha$-adaptin and $G l v-S N A R E$ appears to have a encystation-specific association with $G l$ Rac, which will require further investigation. Here, we have set the scene for future studies to dissect the role of these newly identified components. 
388

389

390

391

392

393

394

395

396

397

398

399

400

401

402

403

404

405

406

407

408

409

410

411

412

413

414

415

416

417

418

419

420

421

422

423

424

425

In summary we have devised a universally applicable ESV staging system based on ESV morphology using CWP1 as a marker. As CWP1 is an easily accessible encystation marker, our staging system can be readily adopted across the field. Beyond morphological differences in ESV stages we have identified molecular markers that can be used to distinguish different stages. As the Giardia toolkit grows, the field will have greater access to tools that allow for functional studies. We anticipate future studies of GlRabs and other ESV-associated proteins identified here that will impact ESV maturation and ESV morphology. Therefore, morphology alone may be insufficient for characterizing/staging the resulting ESVs. The newly identified molecular markers for different ESV stages will be a powerful tool for the purpose of characterizing the stages of abnormal ESVs.

\section{Author Contributions}

ET and AP designed the experiments; ET and RS performed the colocalization with CWP1 analyses; ET and JK performed the affinity purifications. HS performed expression analysis. RJ performed mass spectrometry analysis and analysed the proteomics data. ET and AP wrote the manuscript. MM and AP were responsible for fund acquisition. All authors contributed to the article and approved the submitted version.

\section{Acknowledgments}

This work was supported by NIH Grant 5R01AI1 10708 (A.R.P.) and funding provided by the NIH Yeast Resource Center P41 GM103533 (M.M) supported generation of the mass spectrometry data in part. Authors acknowledge Kelli L. Hvorecny and Melissa Steele-Ogus for their input and critical reading of the manuscript.

\section{References}

Bolte, S., \& Cordelieres, F. P. (2006). A guided tour into subcellular colocalization analysis in light microscopy. Journal of Microscopy, 224(3), 213-232. https://doi.org/10.1111/j.13652818.2006.01706.x

Bolte, S., \& Cordelières, F. P. (2006). A guided tour into subcellular colocalization analysis in light microscopy. In Journal of Microscopy (Vol. 224, Issue 3, pp. 213-232). Blackwell Publishing Ltd. https://doi.org/10.1111/j.1365-2818.2006.01706.x

Boucher, S. E. M., \& Gillin, F. D. (1990). Excystation of in vitro-derived Giardia lamblia cysts. Infection and Immunity, 58(11), 3516-3522. https://doi.org/10.1128/iai.58.11.3516-3522.1990

Chatterjee, A., Carpentieri, A., Ratner, D. M., Bullitt, E., Costello, C. E., Robbins, P. W., \& Samuelson, J. (2010). Giardia cyst wall protein 1 is a lectin that binds to curled fibrils of the GaINAc homopolymer. PLoS Pathogens, 6(8), 61-62. https://doi.org/10.1371/journal.ppat.1001059

Davids, B. J., Mehta, K., Fesus, L., McCaffery, J. M., \& Gillin, F. D. (2004). Dependence of Giardia lamblia encystation on novel transglutaminase activity. Molecular and Biochemical Parasitology, 136(2), 173-180. https://doi.org/10.1016/j.molbiopara.2004.03.011

DuBois, K. N., Abodeely, M., Sakanari, J., Craik, C. S., Lee, M., McKerrow, J. H., \& Sajid, M. 
(2008). Identification of the major cysteine protease of Giardia and its role in encystation. Journal of Biological Chemistry, 283(26), 18024-18031. https://doi.org/10.1074/jbc.M802133200

Eichinger, D. (2001). Encystation in parasitic protozoa. In Current Opinion in Microbiology (Vol. 4, Issue 4, pp. 421-426). Elsevier Ltd. https://doi.org/10.1016/S1369-5274(00)00229-0

Einarsson, E., Troell, K., Hoeppner, M. P., Grabherr, M., Ribacke, U., \& Svärd, S. G. (2016). Coordinated Changes in Gene Expression Throughout Encystation of Giardia intestinalis. 10(3), e0004571. https://doi.org/10.1371/journal.pntd.0004571

Eng, J. K., Jahan, T. A., \& Hoopmann, M. R. (2013). Comet: An open-source MS/MS sequence database search tool. Proteomics, 13(1), 22-24. https://doi.org/10.1002/pmic.201200439

Faso, C., Konrad, C., Schraner, E. M., \& Hehl, A. B. (2013). Export of cyst wall material and Golgi organelle neogenesis in Giardia lamblia depend on endoplasmic reticulum exit sites. Cellular Microbiology, 15(4), 537-553. https://doi.org/10.1111/cmi.12054

Gerwig, G. J., Van Albert Kuik, J., Leeflang, B. R., Kamerling, J. P., Vliegenthart, J. F. G., Karr, C. D., \& Jarroll, E. L. (2002). The Giardia intestinal filamentous cyst wall contains a novel $\beta(1-3)-$ N-acetyl-D-galactosamine polymer: A structural and conformational study. Glycobiology, 12(8), 499-505. https://doi.org/10.1093/glycob/cwf059

Gibson, D. G., Young, L., Chuang, R.-Y., Venter, J. C., Hutchison, C. A., \& Smith, H. O. (2009). Enzymatic assembly of DNA molecules up to several hundred kilobases. Nature Methods, 6(5), 343-345. https://doi.org/10.1038/nmeth.1318

Gourguechon, S., \& Cande, W. Z. (2011). Rapid tagging and integration of genes in Giardia intestinalis. Eukaryotic Cell, 10(1), 142-145. https://doi.org/10.1128/EC.00190-10

Hodge, R. G., \& Ridley, A. J. (2016). Regulating Rho GTPases and their regulators. In Nature Reviews Molecular Cell Biology (Vol. 17, Issue 8, pp. 496-510). Nature Publishing Group. https://doi.org/10.1038/nrm.2016.67

Ioannou, M. S., \& McPherson, P. S. (2016). Regulation of cancer cell behavior by the small GTPase Rab13. In Journal of Biological Chemistry (Vol. 291, Issue 19, pp. 9929-9937). American Society for Biochemistry and Molecular Biology Inc. https://doi.org/10.1074/jbc.R116.715193

Käll, L., Canterbury, J. D., Weston, J., Noble, W. S., \& MacCoss, M. J. (2007). Semi-supervised learning for peptide identification from shotgun proteomics datasets. Nature Methods, 4(11), 923-925. https://doi.org/10.1038/nmeth1113

Konrad, C., Spycher, C., \& Hehl, A. B. (2010). Selective condensation drives partitioning and sequential secretion of cyst wall proteins in differentiating Giardia lamblia. PLoS Pathogens, 6(4), 1-12. https://doi.org/10.1371/journal.ppat.1000835

Krtková, J., Thomas, E. B., Alas, G. C. M., Schraner, E. M., Behjatnia, H. R., Hehl, A. B., \& Paredez, A. R. (2016). Rac Regulates Giardia lamblia Encystation by Coordinating Cyst Wall Protein Trafficking and Secretion. MBio, 7(4). https://doi.org/10.1128/mBio.01003-16 
463

464

465

466

467

468

469

470

471

472

473

474

475

476

477

478

479

480

481

482

483

484

485

486

487

488

489

490

491

492

493

494

495

496

497

498

499

500

501

Lane, S., \& Lloyd, D. (2002). Current trends in research into the waterborne parasite Giardia. In Critical Reviews in Microbiology (Vol. 28, Issue 2, pp. 123-147). CRC Press LLC. https://doi.org/10.1080/1040-840291046713

Lawson, C. D., \& Ridley, A. J. (2018). Rho GTPase signaling complexes in cell migration and invasion. In Journal of Cell Biology (Vol. 217, Issue 2, pp. 447-457). Rockefeller University Press. https://doi.org/10.1083/jcb.201612069

Li, G., \& Marlin, M. C. (2015). Rab family of GTpases. Methods in Molecular Biology, 1298, 1. https://doi.org/10.1007/978-1-4939-2569-8_1

Luján, H. D., Mowatt, M. R., Byrd, L. G., \& Nash, T. E. (1996). Cholesterol starvation induces differentiation of the intestinal parasite Giardia lamblia. Proceedings of the National Academy of Sciences of the United States of America, 93(15), 7628-7633. https://doi.org/10.1073/pnas.93.15.7628

Lujan, H. D., Mowatt, M. R., Conrad, J. T., Bowers, B., \& Nash, T. E. (1995). Identification of a novel Giardia lamblia cyst wall protein with leucine- rich repeats: Implications for secretory granule formation and protein assembly into the cyst wall. Journal of Biological Chemistry, 270(49), 29307-29313. https://doi.org/10.1074/jbc.270.49.29307

Mani, S., \& Thattai, M. (2016). Stacking the odds for golgi cisternal maturation. ELife, 5(AUGUST). https://doi.org/10.7554/eLife.16231

Manser, E., Leung, T., Salihuddin, H., Zhao, Z. S., \& Lim, L. (1994). A brain serine/threonine protein kinase activated by Cdc42 and Rac1. Nature, 367(6458), 40-46. https://doi.org/10.1038/367040a0

Marti, M., Li, Y., Schraner, E. M., Wild, P., Köhler, P., \& Hehl, A. B. (2003). The secretory apparatus of an ancient eukaryote: Protein sorting to separate export pathways occurs before formation of transient Golgi-like compartments. Molecular Biology of the Cell, 14(4), 14331447. https://doi.org/10.1091/mbc.E02-08-0467

Michaels, S. A., Shih, H.-W., Zhang, B., Navaluna, E. D., Zhang, Z., Ranade, R. M., Gillespie, J. R., Merritt, E. A., Fan, E., Buckner, F. S., Paredez, A. R., \& Ojo, K. K. (2020). Methionyl-tRNA synthetase inhibitor has potent in vivo activity in a novel Giardia lamblia luciferase murine infection model. The Journal of Antimicrobial Chemotherapy, 75(5), 1218-1227. https://doi.org/10.1093/jac/dkz567

Minamino, N., \& Ueda, T. (2019). RAB GTPases and their effectors in plant endosomal transport. In Current Opinion in Plant Biology (Vol. 52, pp. 61-68). Elsevier Ltd. https://doi.org/10.1016/j.pbi.2019.07.007

Paredez, A. R., Nayeri, A., Xu, J. W., Krtková, J., Cande, W. Z., \& Zacheus Cande, W. (2014). Identification of obscure yet conserved actin-associated proteins in Giardia lamblia. Eukaryotic Cell, 13(6), 776-784. https://doi.org/10.1128/EC.00041-14

Pfeffer, S. R. (2017). Rab GTPases: Master regulators that establish the secretory and endocytic pathways. In Molecular Biology of the Cell (Vol. 28, Issue 6, pp. 712-715). American Society for Cell Biology. https://doi.org/10.1091/mbc.E16-10-0737 
502

503

504

505

506

507

508

509

510

511

512

513

514

515

516

517

518

519

520

521

522

523

524

525

526

527

528

529

530

531

532

533

534

535

536

537

538

539

540

Pham, J. K., Nosala, C., Scott, E. Y., Nguyen, K. F., Hagen, K. D., Starcevich, H. N., \& Dawson, C. (2017). Transcriptomic Profiling of High-Density Giardia Foci Encysting in the Murine Proximal Intestine. Frontiers in Cellular and Infection Microbiology, 7(MAY), 227. https://doi.org/10.3389/fcimb.2017.00227

Phuyal, S., \& Farhan, H. (2019). Multifaceted Rho GTPase Signaling at the Endomembranes. Frontiers in Cell and Developmental Biology, 7, 127. https://doi.org/10.3389/fcell.2019.00127

Reiner, D. S., Ankarklev, J., Troell, K., Palm, D., Bernander, R., Gillin, F. D., Andersson, J. O., \& Svärd, S. G. (2008). Synchronisation of Giardia lamblia: Identification of cell cycle stagespecific genes and a differentiation restriction point. International Journal for Parasitology, 38(8-9), 935-944. https://doi.org/10.1016/j.ijpara.2007.12.005

Reiner, D. S., McCaffery, J. M., \& Gillin, F. D. (2001). Reversible interruption of Giardia lamblia cyst wall protein transport in a novel regulated secretory pathway. Cellular Microbiology, 3(7), 459-472. https://doi.org/10.1046/j.1462-5822.2001.00129.x

Saraste, J. (2016). Spatial and functional aspects of ER-Golgi Rabs and Tethers. Frontiers in Cell and Developmental Biology, 4(APR), 28. https://doi.org/10.3389/fcell.2016.00028

Schindelin, J., Arganda-Carreras, I., Frise, E., Kaynig, V., Longair, M., Pietzsch, T., Preibisch, S., Rueden, C., Saalfeld, S., Schmid, B., Tinevez, J.-Y., White, D. J., Hartenstein, V., Eliceiri, K., Tomancak, P., \& Cardona, A. (2012). Fiji: an open-source platform for biological-image analysis. Nature Methods, 9(7), 676-682. https://doi.org/10.1038/nmeth.2019

Slavin, I., Saura, A., Carranza, P. G., Touz, M. C., Nores, M. J., \& Luján, H. D. (2002). Dephosphorylation of cyst wall proteins by a secreted lysosomal acid phosphatase is essential for excystation of Giardia lamblia. Molecular and Biochemical Parasitology, 122(1), 95-98. https://doi.org/10.1016/S0166-6851(02)00065-8

Srinivasan, S., Wang, F., Glavas, S., Ott, A., Hofmann, F., Aktories, K., Kalman, D., \& Bourne, H. R. (2003). Rac and Cdc42 play distinct roles in regulating PI(3,4,5)P3 and polarity during neutrophil chemotaxis. Journal of Cell Biology, 160(3), 375-385. https://doi.org/10.1083/jcb.200208179

Štefanić, S., Morf, L., Kulangara, C., Regös, A., Sonda, S., Schraner, E., Spycher, C., Wild, P., \& Hehl, A. B. (2009). Neogenesis and maturation of transient Golgi-like cisternae in a simple eukaryote. Journal of Cell Science, 122(16), 2846-2856. https://doi.org/10.1242/jcs.049411

Sun, C. H., McCaffery, J. M., Reiner, D. S., \& Gillin, F. D. (2003). Mining the Giardia lamblia genome for new cyst wall proteins. Journal of Biological Chemistry, 278(24), 21701-21708. https://doi.org/10.1074/jbc.M302023200

Touz, María C., Nores, M. J., Slavin, I., Carmona, C., Conrad, J. T., Mowatt, M. R., Nash, T. E., Coronel, C. E., \& Luján, H. D. (2002). The activity of a developmentally regulated cysteine proteinase is required for cyst wall formation in the primitive eukaryote Giardia lamblia. Journal of Biological Chemistry, 277(10), 8474-8481. https://doi.org/10.1074/jbc.M110250200

Touz, Maria C., \& Zamponi, N. (2017). Sorting without a Golgi complex. In Traffic (Vol. 18, Issue 10, pp. 637-645). Blackwell Munksgaard. https://doi.org/10.1111/tra.12500 
541 Vernoud, V., Horton, A. C., Yang, Z., \& Nielsen, E. (2003). Analysis of the small GTPase gene superfamily of arabidopsis. Plant Physiology, 131(3), 1191-1208. https://doi.org/10.1104/pp.013052

Wiśniewski, J. R., Zougman, A., Nagaraj, N., \& Mann, M. (2009). Universal sample preparation method for proteome analysis. Nature Methods, 6(5), 359-362. https://doi.org/10.1038/nmeth.1322

\section{Tables}

548 Table 1 List of candidate genes selected for this study and identified as hits in OS-GlRac pulldown

549 experiment in trophozoites and encysting cells ( $8 \mathrm{~h}$ p.i.e.) that are homologs of known membrane 550 trafficking players.

\begin{tabular}{|c|c|c|c|c|c|c|c|}
\hline \multirow{2}{*}{ Gene ID } & \multirow{2}{*}{ Gene Name } & \multicolumn{3}{|c|}{ Trophozoites } & \multicolumn{3}{|c|}{ 8hr encyst } \\
\hline & & $\begin{array}{c}\text { Replicate } \\
1\end{array}$ & $\begin{array}{c}\text { Replicate } \\
2\end{array}$ & $\begin{array}{c}\text { Replicate } \\
3\end{array}$ & $\begin{array}{c}\text { Replicate } \\
1\end{array}$ & $\begin{array}{c}\text { Replicate } \\
2\end{array}$ & $\begin{array}{c}\text { Replicate } \\
3\end{array}$ \\
\hline GL50803_8496 & $\begin{array}{l}\text { Rac/Rho- } \\
\text { like protein }\end{array}$ & $\mathbf{x}$ & $\mathbf{x}$ & $\mathbf{x}$ & $\mathbf{x}$ & $\mathbf{x}$ & $\mathbf{x}$ \\
\hline GL50803_5744 & Sec61- $\alpha$ & $\mathrm{x}$ & $\mathrm{x}$ & $\mathrm{x}$ & $\mathrm{x}$ & $\mathrm{x}$ & $\mathrm{x}$ \\
\hline GL50803_9558 & Rab1a & & $\mathrm{x}$ & $\mathrm{x}$ & $\mathrm{x}$ & $\mathrm{x}$ & $\mathrm{x}$ \\
\hline GL50803_15567 & Rab2a & & $\mathrm{x}$ & $\mathrm{x}$ & $\mathrm{x}$ & $\mathrm{x}$ & $\mathrm{x}$ \\
\hline GL50803_11953 & $\begin{array}{l}\text { Coatomer } \alpha \\
\text { subunit }\end{array}$ & & $\mathrm{x}$ & $\mathrm{x}$ & & $\mathrm{x}$ & $\mathrm{x}$ \\
\hline GL50803_9593 & $\begin{array}{l}\text { Coatomer } \beta^{\prime} \\
\text { subunit }\end{array}$ & & $\mathrm{x}$ & $\mathrm{x}$ & & $\mathrm{x}$ & $\mathrm{x}$ \\
\hline GL50803_17304 & Alpha $\alpha$ & & & & & $\mathrm{x}$ & \\
\hline GL50803_114776 & NSF & & & & & $\mathrm{x}$ & \\
\hline GL50803_9489 & v-SNARE & & & & & $\mathrm{x}$ & \\
\hline
\end{tabular}

\section{$551 \quad$ Figures :}



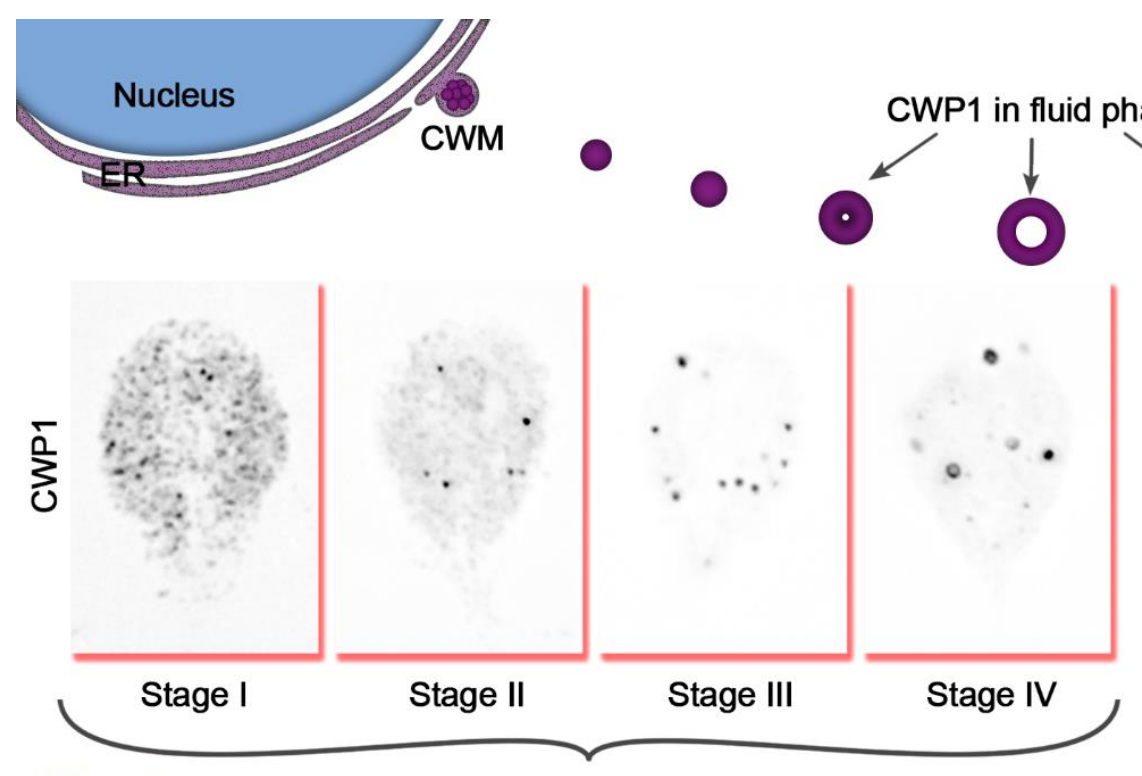

Fig. 1

Found at $8 \mathrm{~h}$ p.i.e

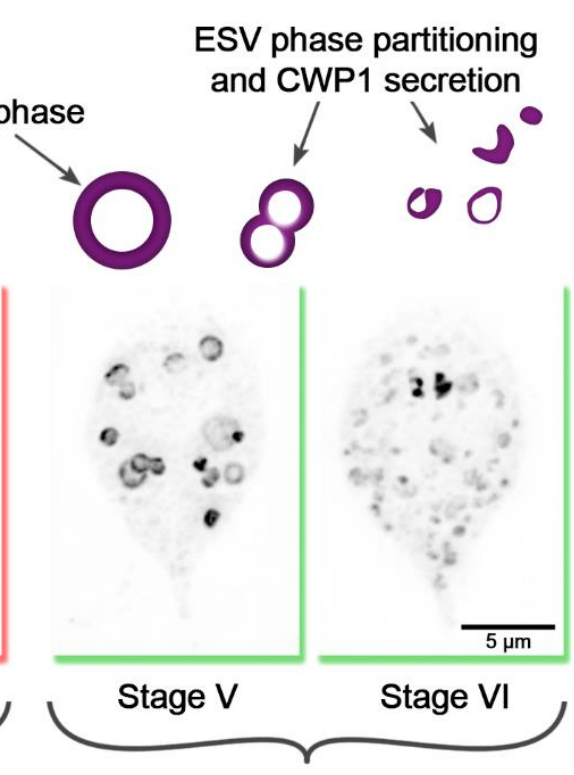

Found at $24 \mathrm{~h}$ p.i.e.

Fig. 1 - Cyst wall protein trafficking in ESVs during encystation can be divided into stages. Graphic showing trafficking of CWP1-containing ESVs. Once Giardia cells sense the signal to encyst, large amounts of CWM are produced in the ER (Stage I), which then accumulate at ER exit sites (Stage II) and are secreted out into the cytoplasm compartmentalized in ESVs (Stage III). As CWP cargo are sorted and processed, they are separated into phases with CWP1 now being confined to the outer fluid-phase of the ESVs (Stage IV) which grow larger in size as they continue to mature (Stage V). The distinct phases of ESVs are then partitioned into separate vesicles (Stage VI) which are subsequently sequentially secreted to form Giardia's cyst wall. These stages can be tracked by visualizing CWP1 and tracking ESV morphology. Cells shown here were harvested and fixed at $8 \mathrm{~h}$ p.i.e. and $24 \mathrm{~h}$ p.i.e., then stained with CWP1 antibody. 


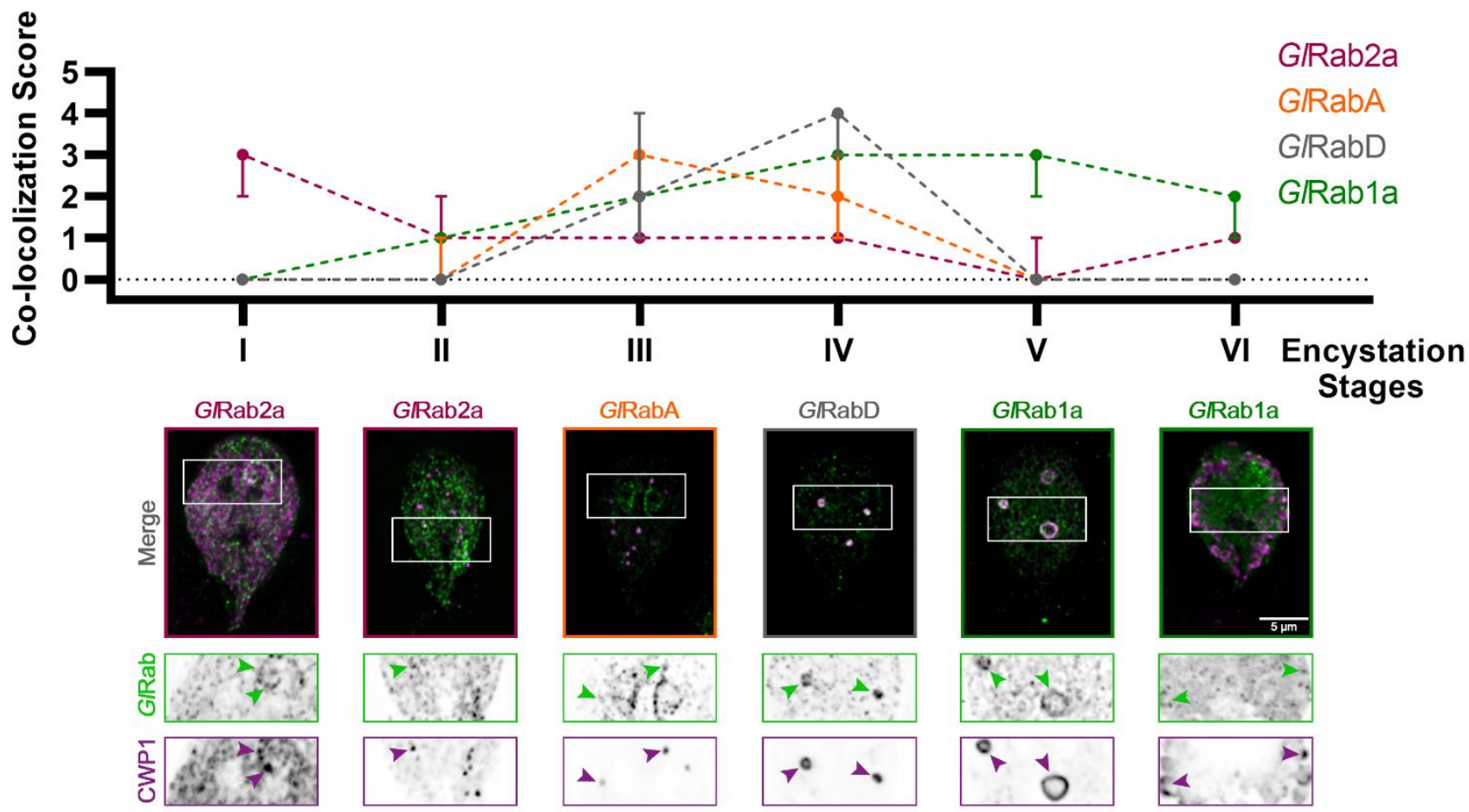

Fig. 2 - Giardia Rabs associate with ESVs during encystation in a stage-specific manner. Summary of findings from colocalization analysis of Giardia Rabs and CWP1 through all the encystation stages described above. Cells expressing endogenously tagged $\mathrm{mNG}-G l$ Rabs were subjected to the two-step encystation process. They were then harvested at $8 \mathrm{~h}$ and $24 \mathrm{~h}$ p.i.e. to be fixed and stained for CWP1. 15-20 cells per encystation stage were then imaged to visualize mNG-tagged GlRabs (green) and CWP1 (magenta) and scored for the level of colocalization between the tagged GlRabs and CWP1 stained structures. Plot shows median scores with 95\% confidence interval. Arrowheads indicate mNG-GlRabs colocalizing with CWP1-stained ESVs. 

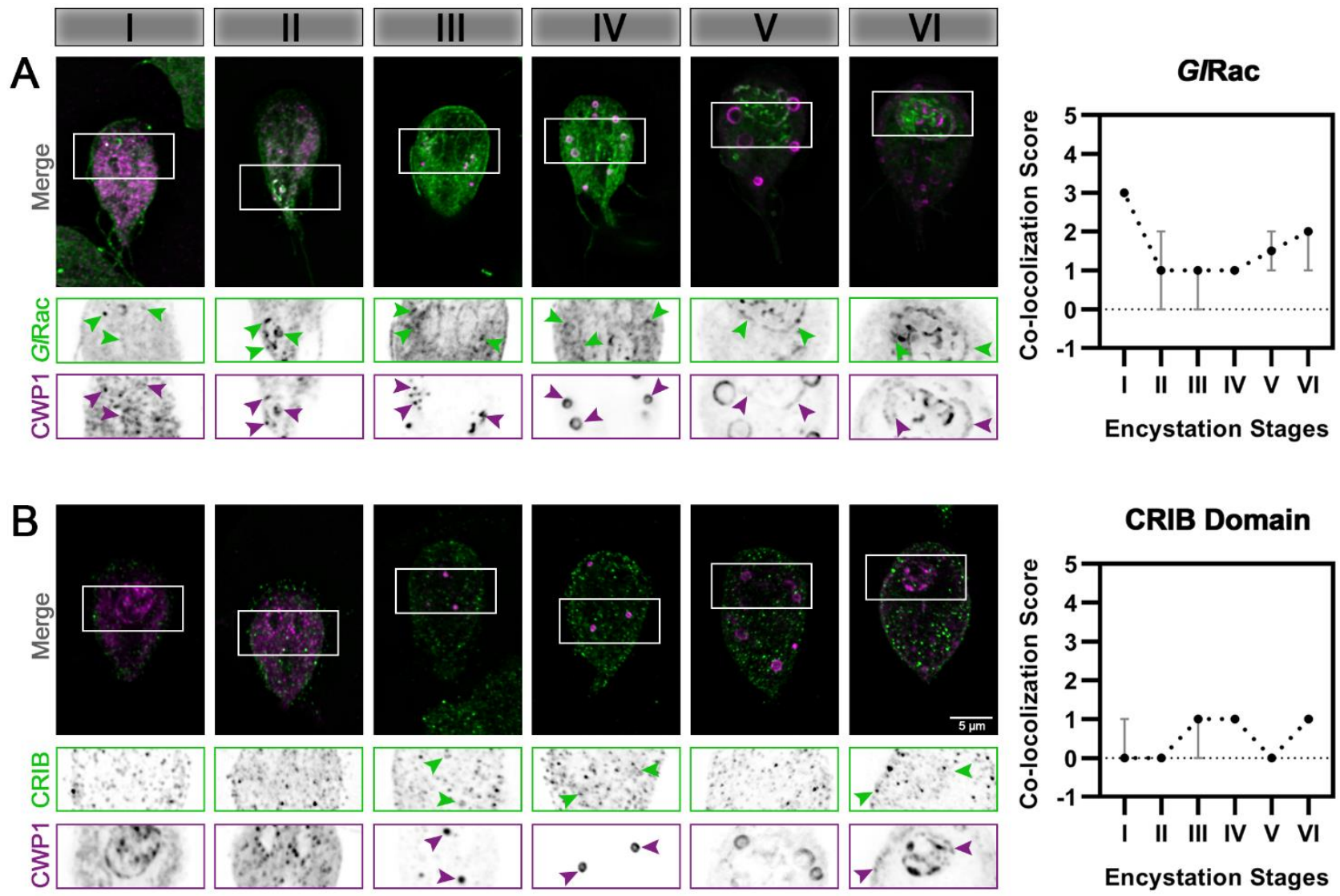

Fig. 3-GlRac activity during encystation is stage-specific. Cells expressing HALO-GlRac (endogenous tag) or CRIB-HA-mNG (exogenous expression) were subjected to the two-step encystation process. They were then harvested at $8 \mathrm{~h}$ and $24 \mathrm{~h}$ p.i.e. to be fixed and stained for HALOGlRac or CRIB-HA-mNG and CWP1. 15-20 cells per encystation stage were then imaged to visualize HALO-GlRac (green) or CRIB-HA-mNG (green) and CWP1 (magenta) and scored for the level of colocalization between HALO-GlRac or CRIB-HA-mNG and CWP1 stained structures. Plot shows median scores with 95\% confidence interval. Arrowheads indicate Halo-GlRac or CRIB-HAmNG colocalizing with CWP1-stained ESVs. 


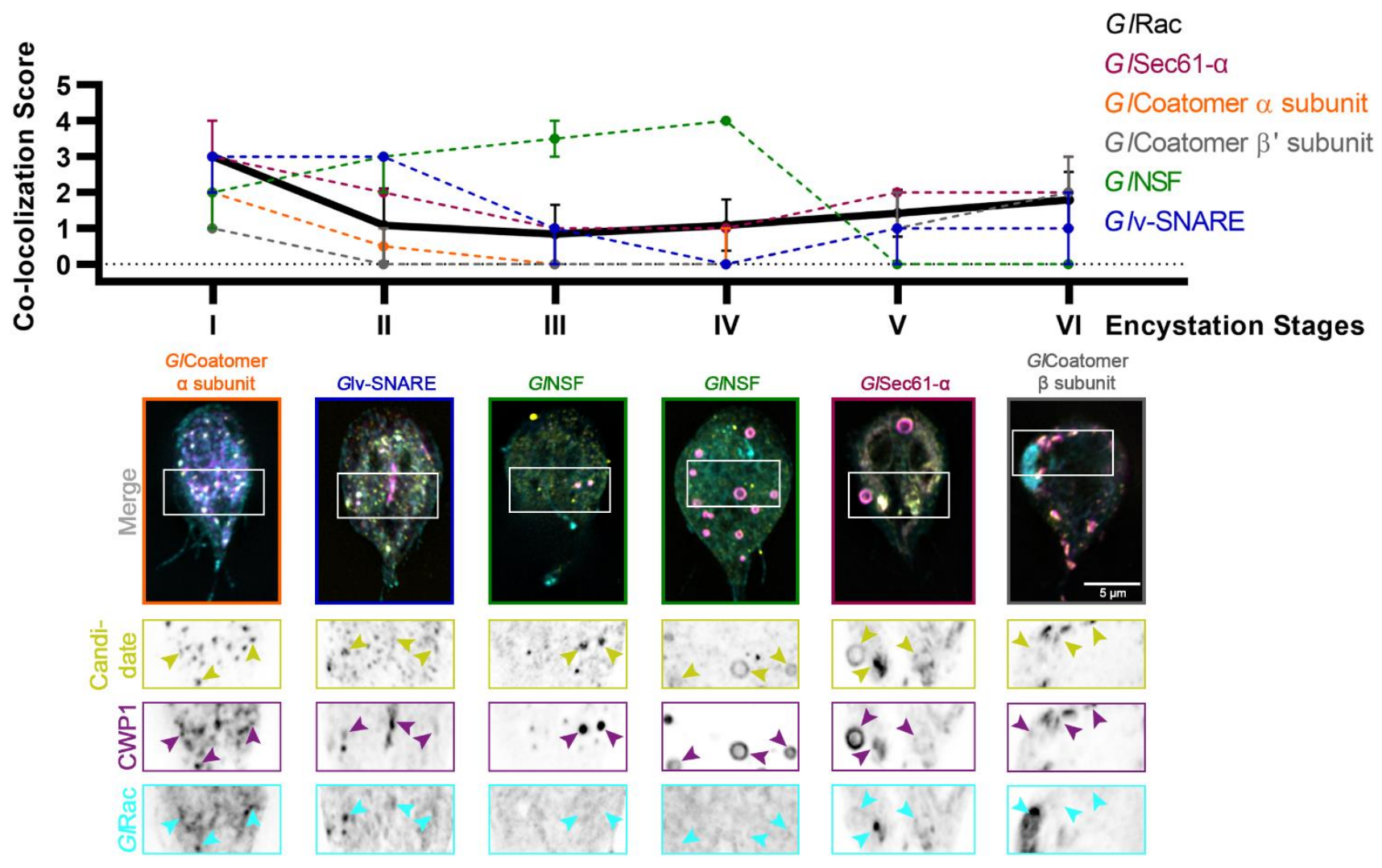

584 Fig. 4 - Putative effectors of GlRac colocalize with CWP1 in a stage-specific manner in a pattern 585 that is similar to GlRac. Summary of findings from colocalization analysis of putative GlRac 586 interactors and CWP1 through all the encystation stages described above. Cells expressing endogenously tagged HALO-GlRac and mNG-HA tagged candidates were subjected to the two-step encystation process. They were then harvested at $8 \mathrm{~h}$ and $24 \mathrm{~h}$ p.i.e. to be fixed and stained for CWP1. 15-20 cells per encystation stage were then imaged to visualize HALO-GlRac (cyan) or HA-mNGcandidate/candidate-HA-mNG (yellow) and CWP1 (magenta) and scored for the level of colocalization between the tagged candidate and CWP1 stained structures. Plot shows median scores with $95 \%$ confidence interval. Arrowheads indicate candidate proteins colocalizing with CWP1stained ESVs. 


\section{A pCWP1-Eluc_ThreeEncystTime}

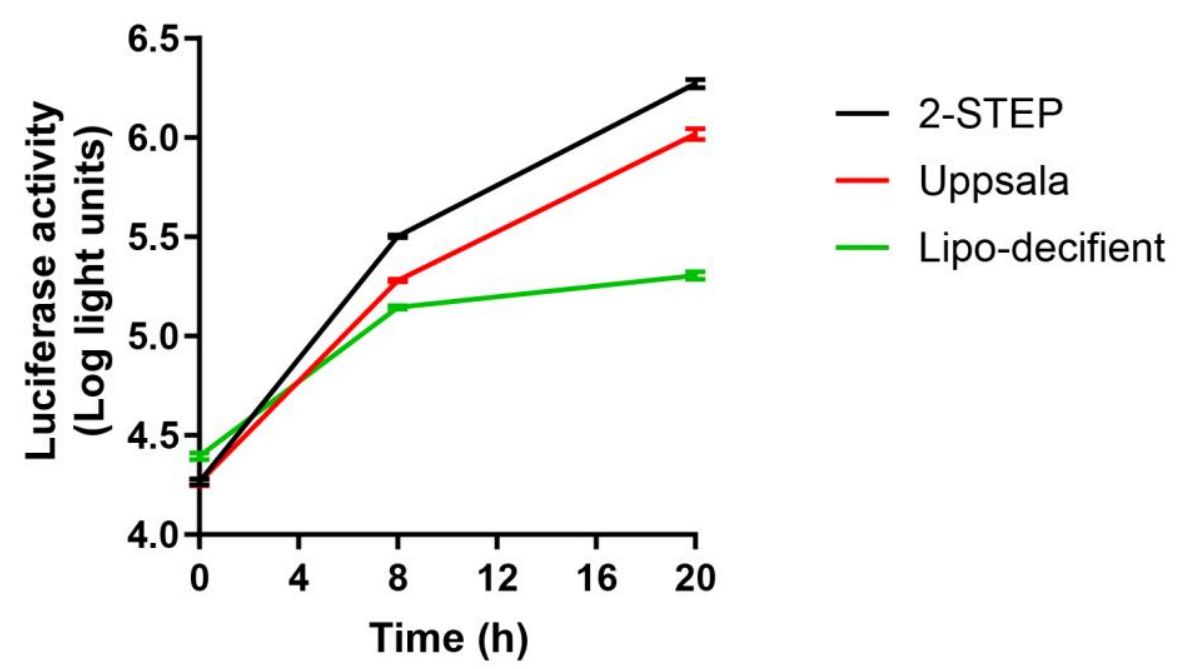

B

pCWP2-Eluc_ThreeEncyst

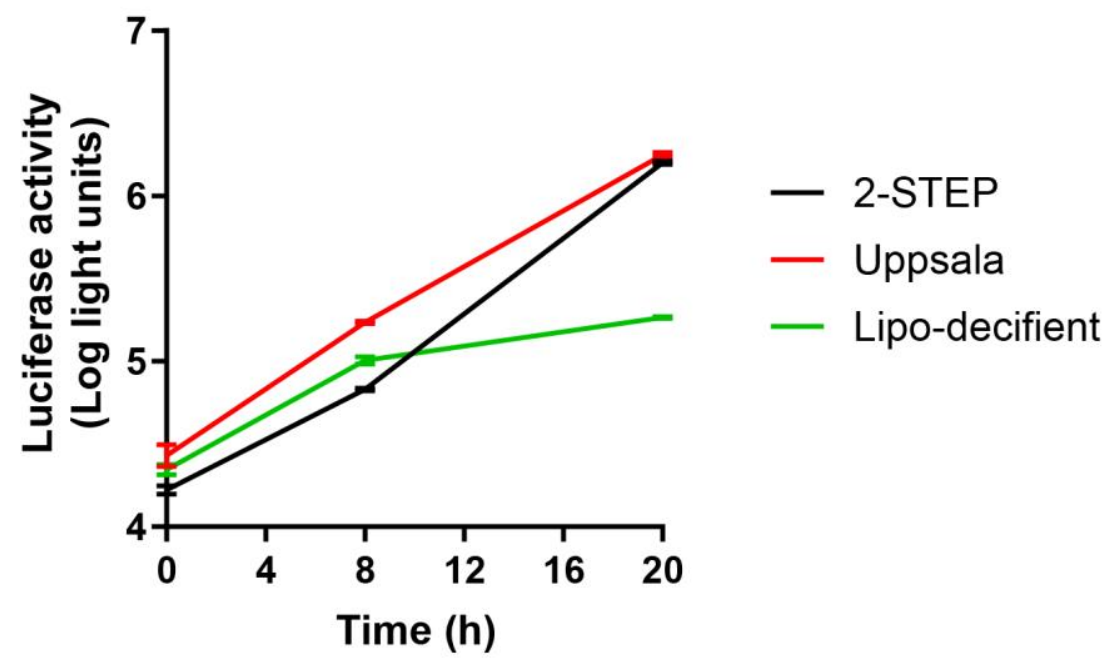

Fig. S1 Comparison of CWP1-Eluc and CWP2-Eluc reporter strains using three common methods to induce encystation in Giardia. This is a single experiment with three separate technical replicates for each condition and timepoint. While the CWP1 result for Two-Step and Uppsala appear similar in this bulk assay, it should be noted that the high bile levels used in the Uppsala method kill off a portion of the parasites and this results in a stronger induction of encystation for the surviving cells. 


\section{G/Rab2a}

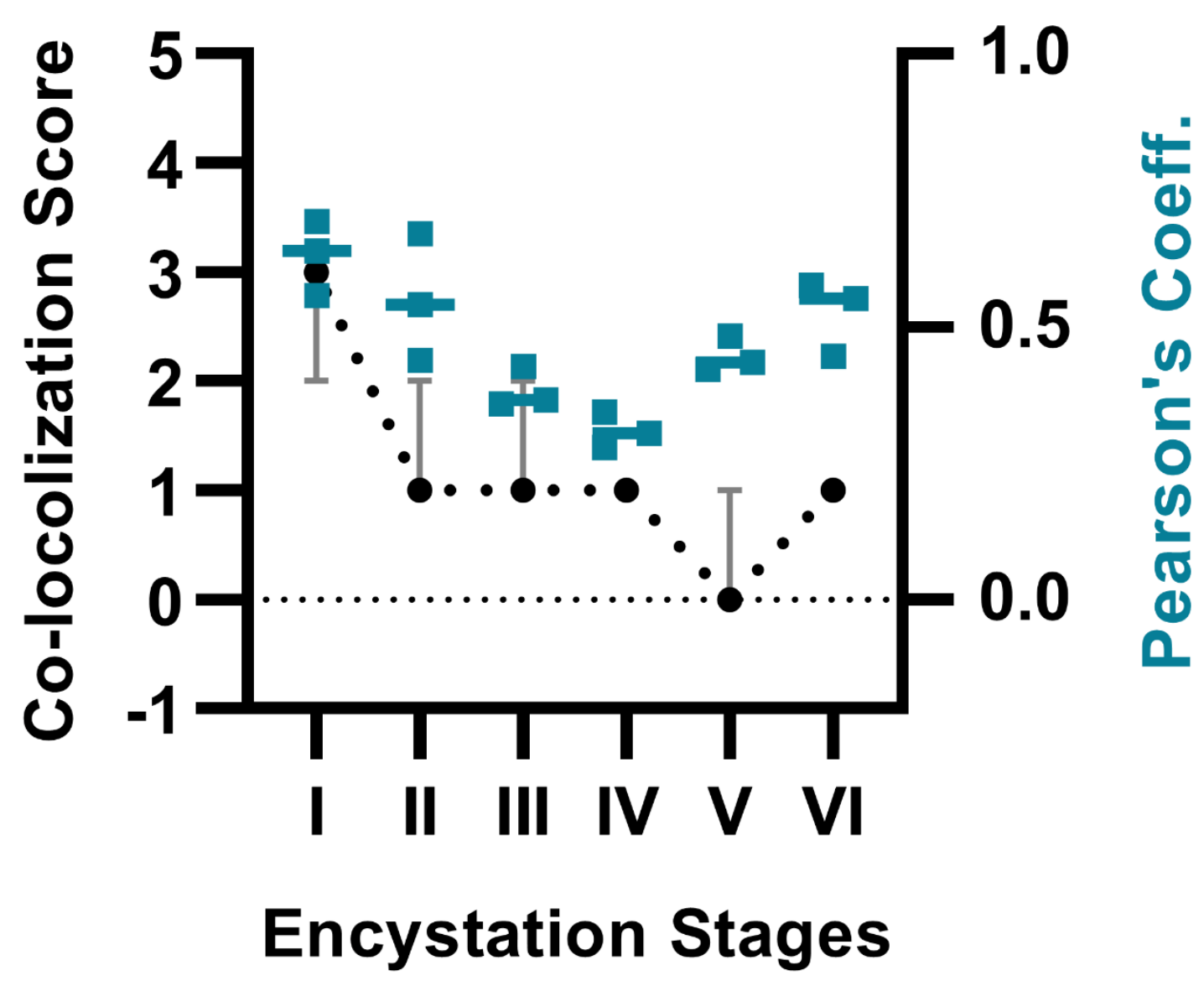

Fig. S2 Comparing manual scores and Pearson's

Correlation Co-efficients for colocalization levels between

GIRab2a and CWP1 across the 6 encystation stages.

Plot shows median scores with $95 \%$ confidence interval. 

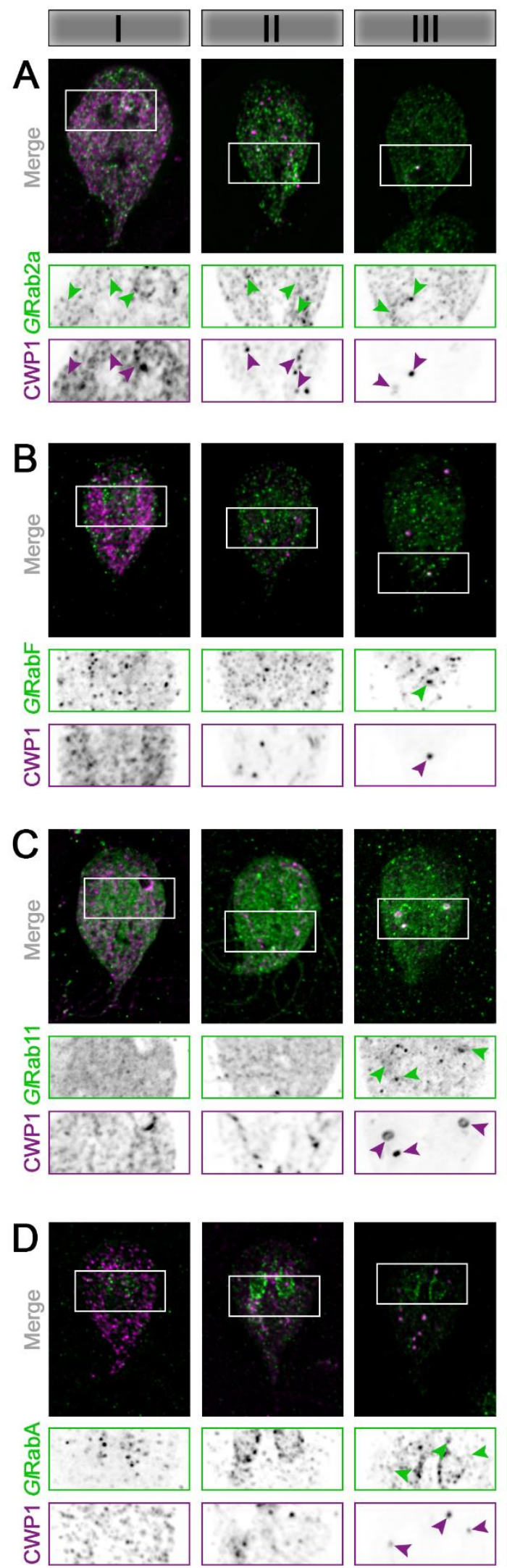
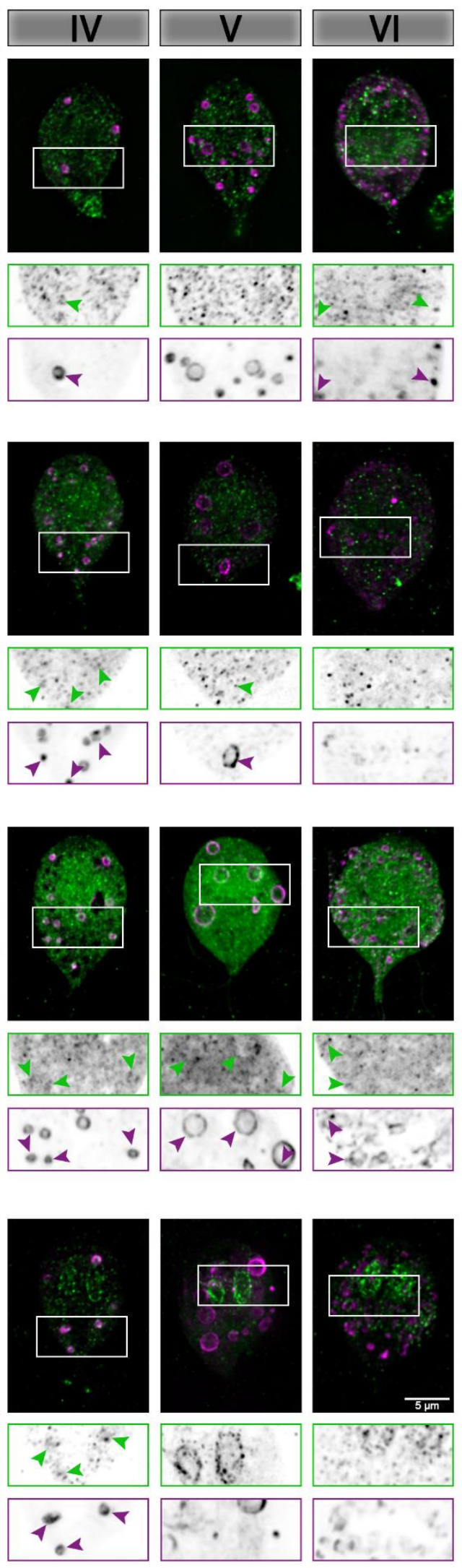

G/Rab2a

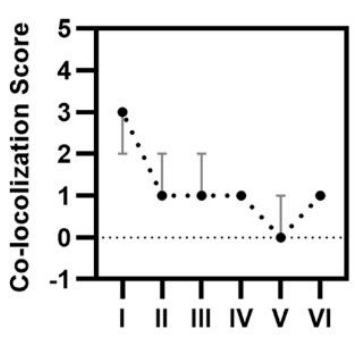

Encystation Stages

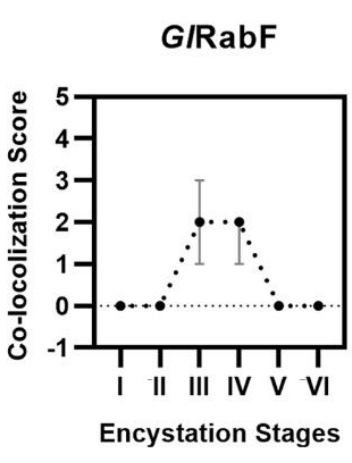

GIRab11

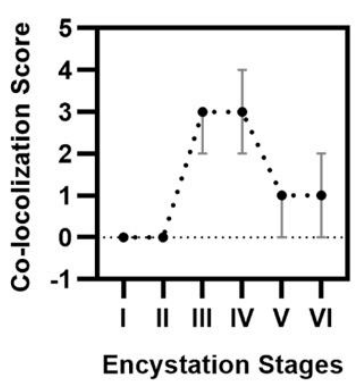

GIRabA

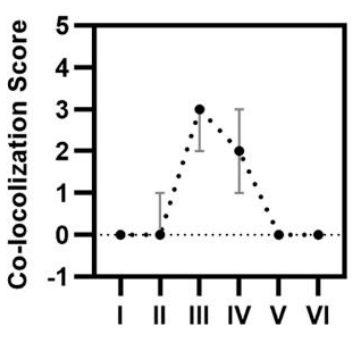

Encystation Stages 

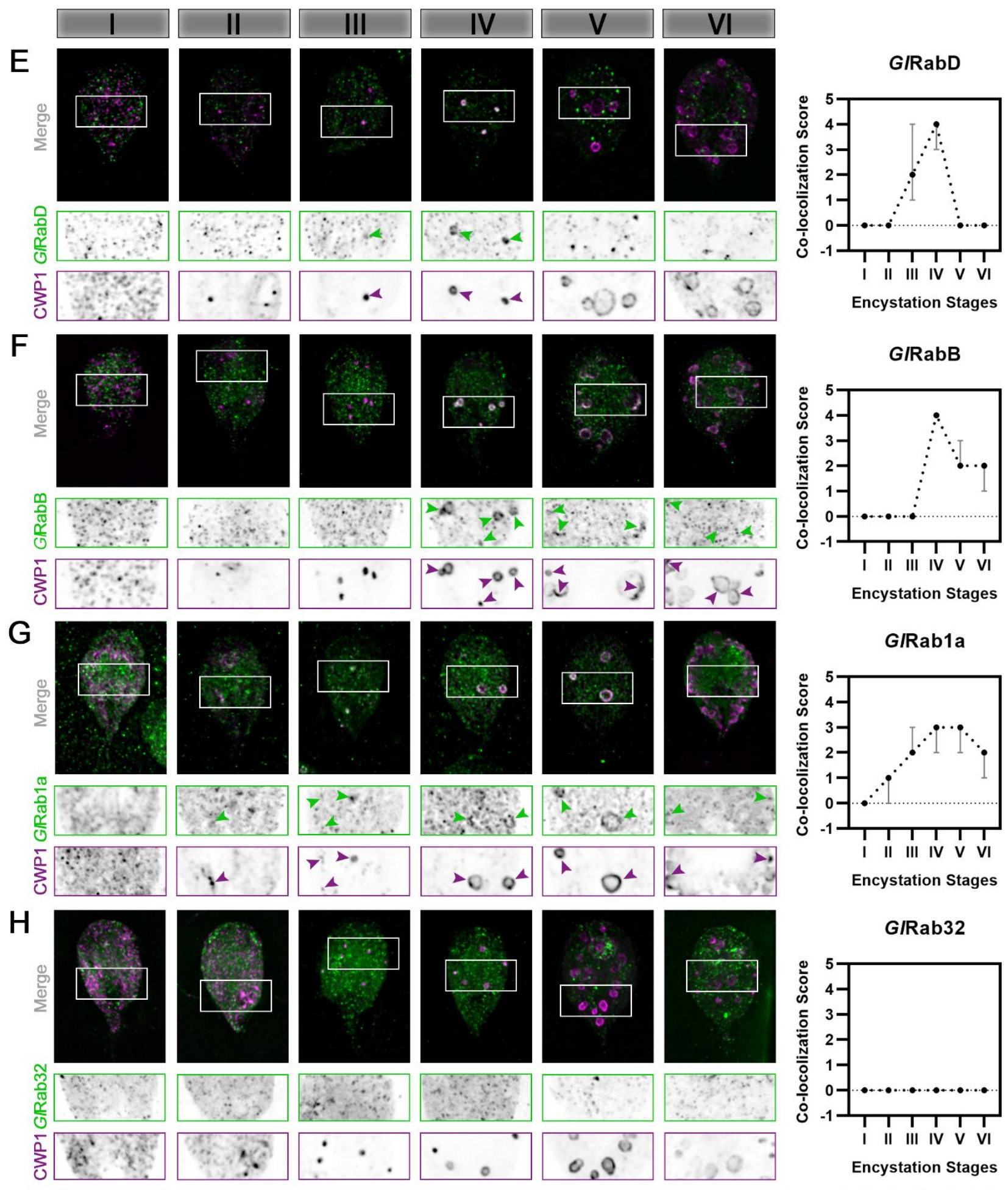

GIRabB

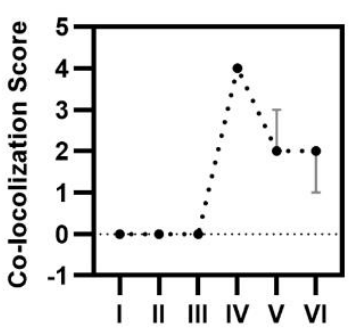

Encystation Stages

GIRab1a

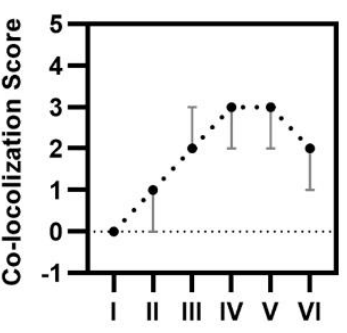

Encystation Stages

G/Rab32

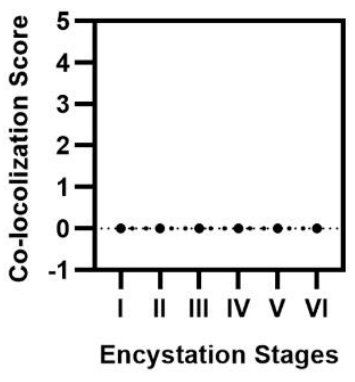

Fig. S3 Giardia Rabs associate with ESVs during encystation in a stage-specific manner. Colocalization analysis of Giardia Rabs and CWP1 through the encystation stages. Cells expressing endogenously tagged mNG-G/Rabs were subjected to the two-step encystation process. They were then harvested at $8 \mathrm{~h}$ and $24 \mathrm{~h}$ p.i.e to be fixed and stained for CWP1. 15-20 cells per encystation stage were then imaged to visualize mNG-tagged GIRabs (green) and CWP1 (magenta) and scored for the level of colocalization between the tagged GIRabs and CWP1 stained structures. Plots show median scores with $95 \%$ confidence interval. Arrowheads indicate mNG-GIRabs colocalizing with CWP1-stained ESVs. 


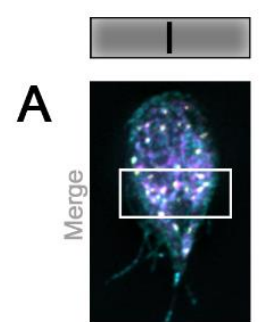

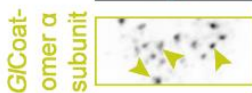

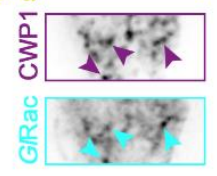

B

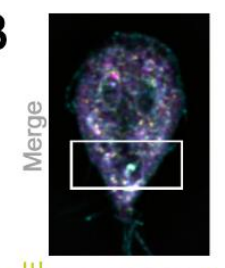

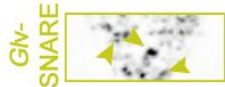

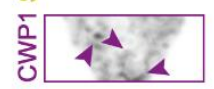

is alc

C

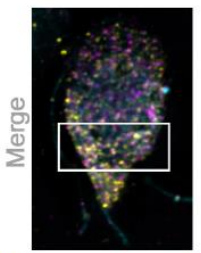

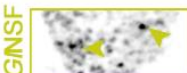

$\sum_{0}^{5} e^{0}<*$

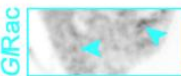

D

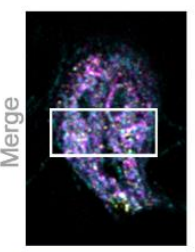

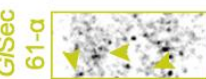

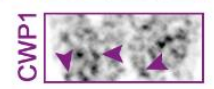

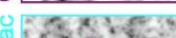

601

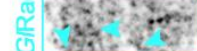

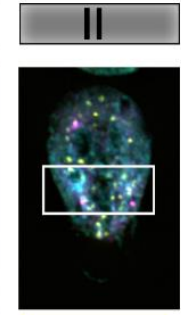

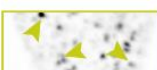

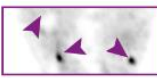

र2
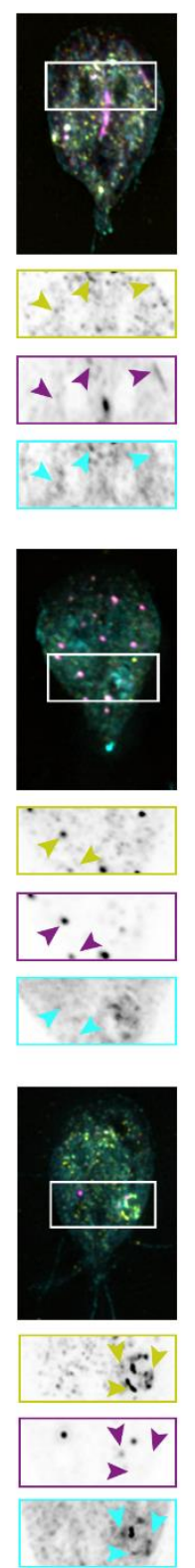
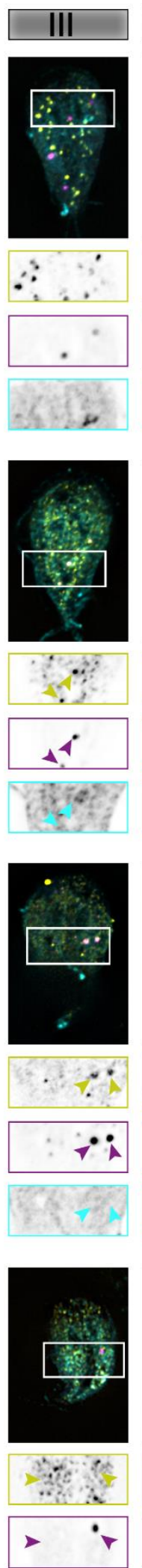

क्षे
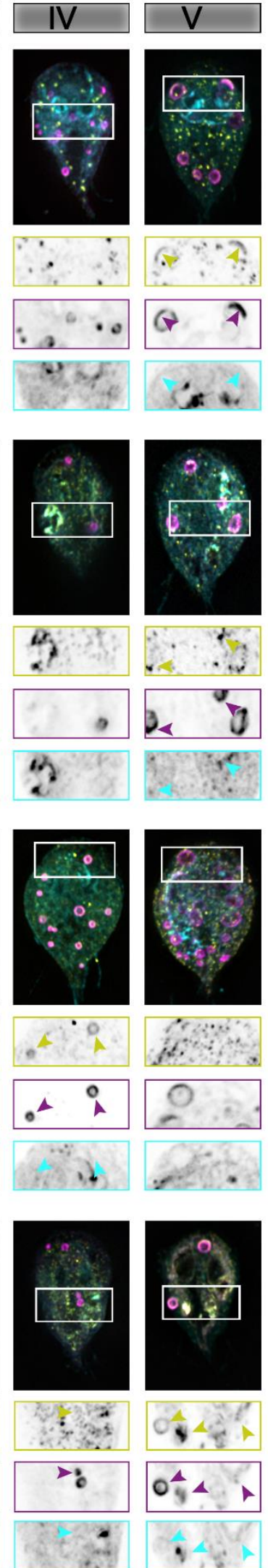
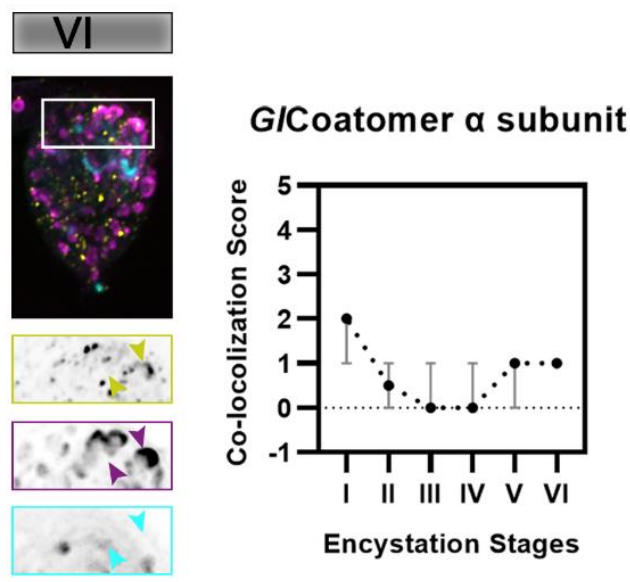

Encystation Stages
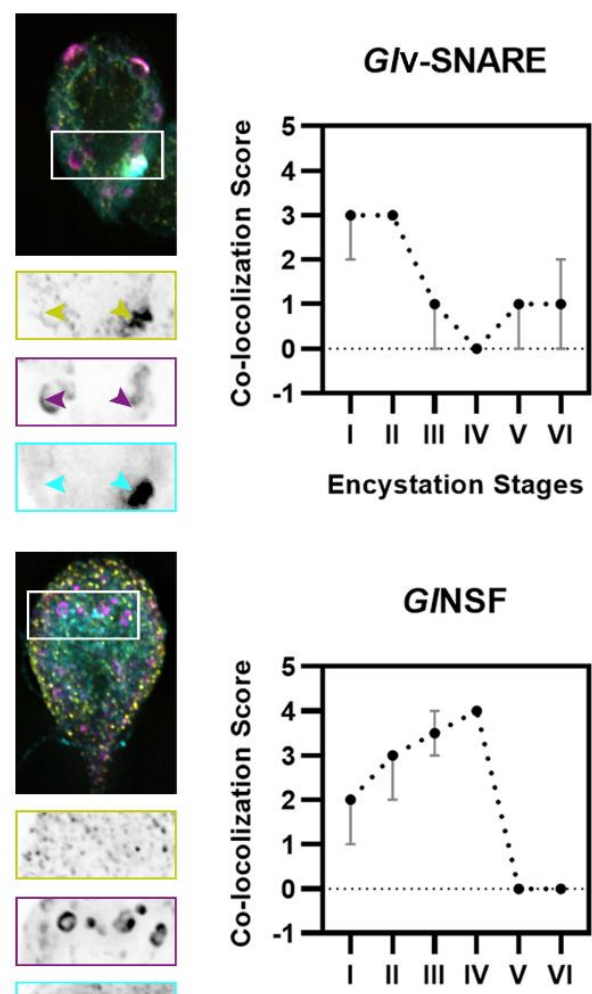

Encystation Stages

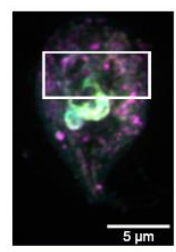

GISec61- $\alpha$

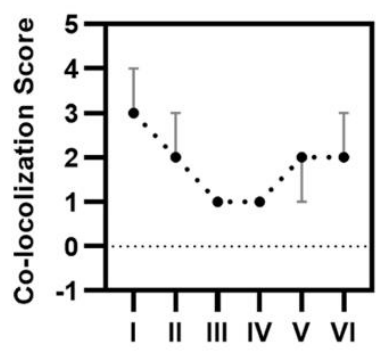

Encystation Stages 


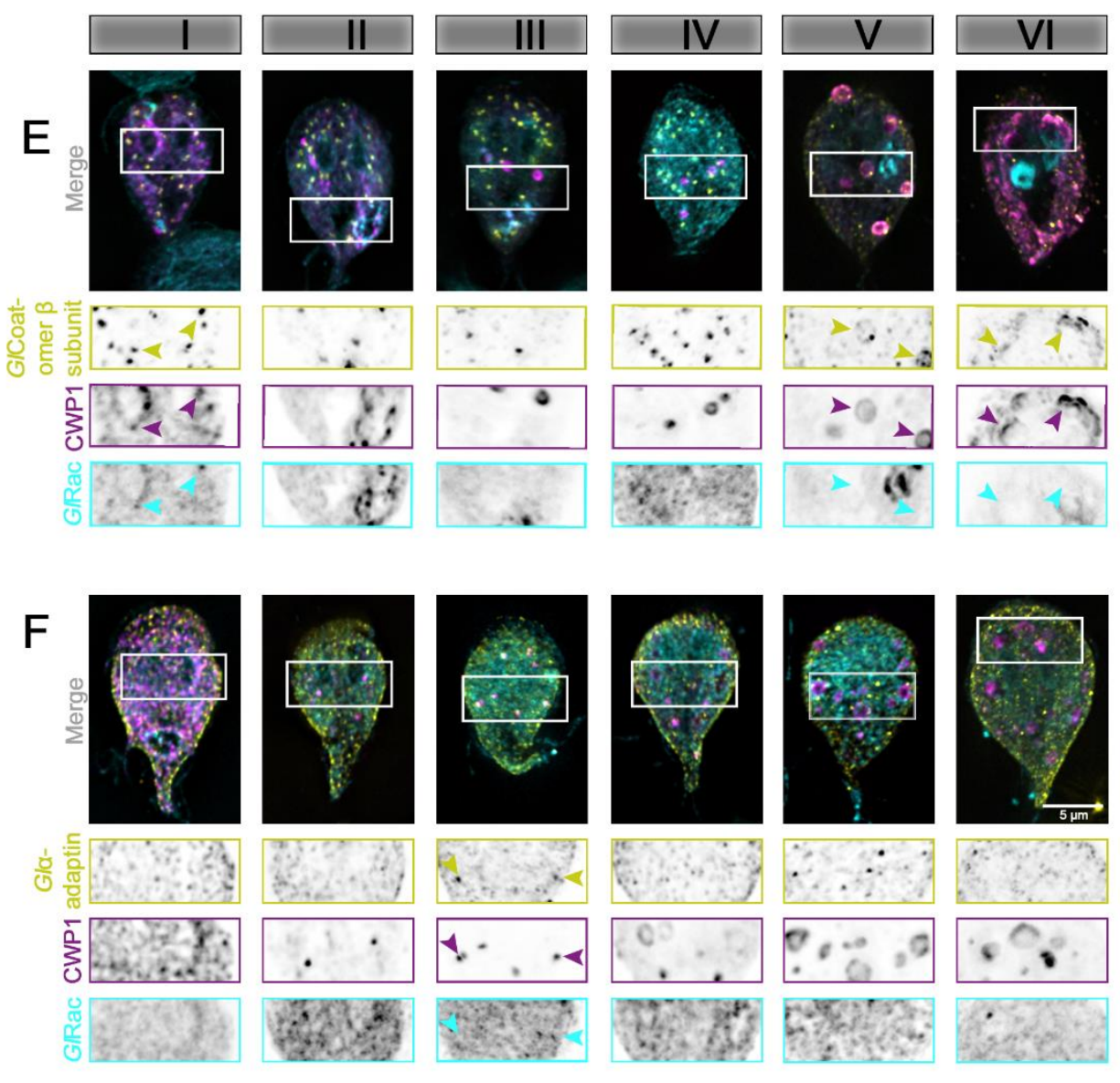

\section{GICoatomer $\beta$ ' subunit}
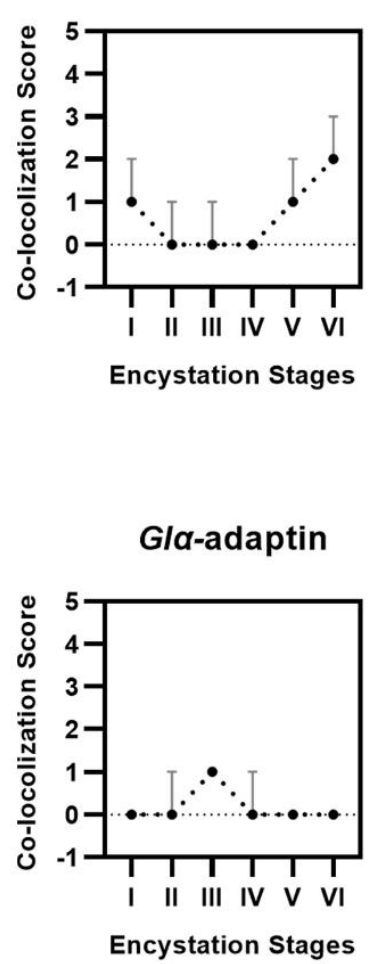

Fig. S4 Putative effectors of GIRac colocalize with CWP1 in a stage-specific manner. Colocalization analysis of putative GIRac interactors and CWP1 through all the encystation stages described above. Cells expressing endogenously tagged HALO-GIRac and mNG-HA tagged candidates were subjected to the two-step encystation process. They were then harvested at $8 \mathrm{~h}$ and $24 \mathrm{~h}$ p.i.e. to be fixed and stained for CWP1. 15-20 cells per encystation stage were then imaged to visualize HALO-GIRac (cyan) or HA-mNG-candidate/ candidate-HA-mNG (yellow) and CWP1 (magenta) and scored for the level of colocalization between the tagged candidate and CWP1 stained structures. Plot shows median scores with $95 \%$ confidence interval. Arrowheads indicate candidates colocalizing with CWP1-stained ESVs. 

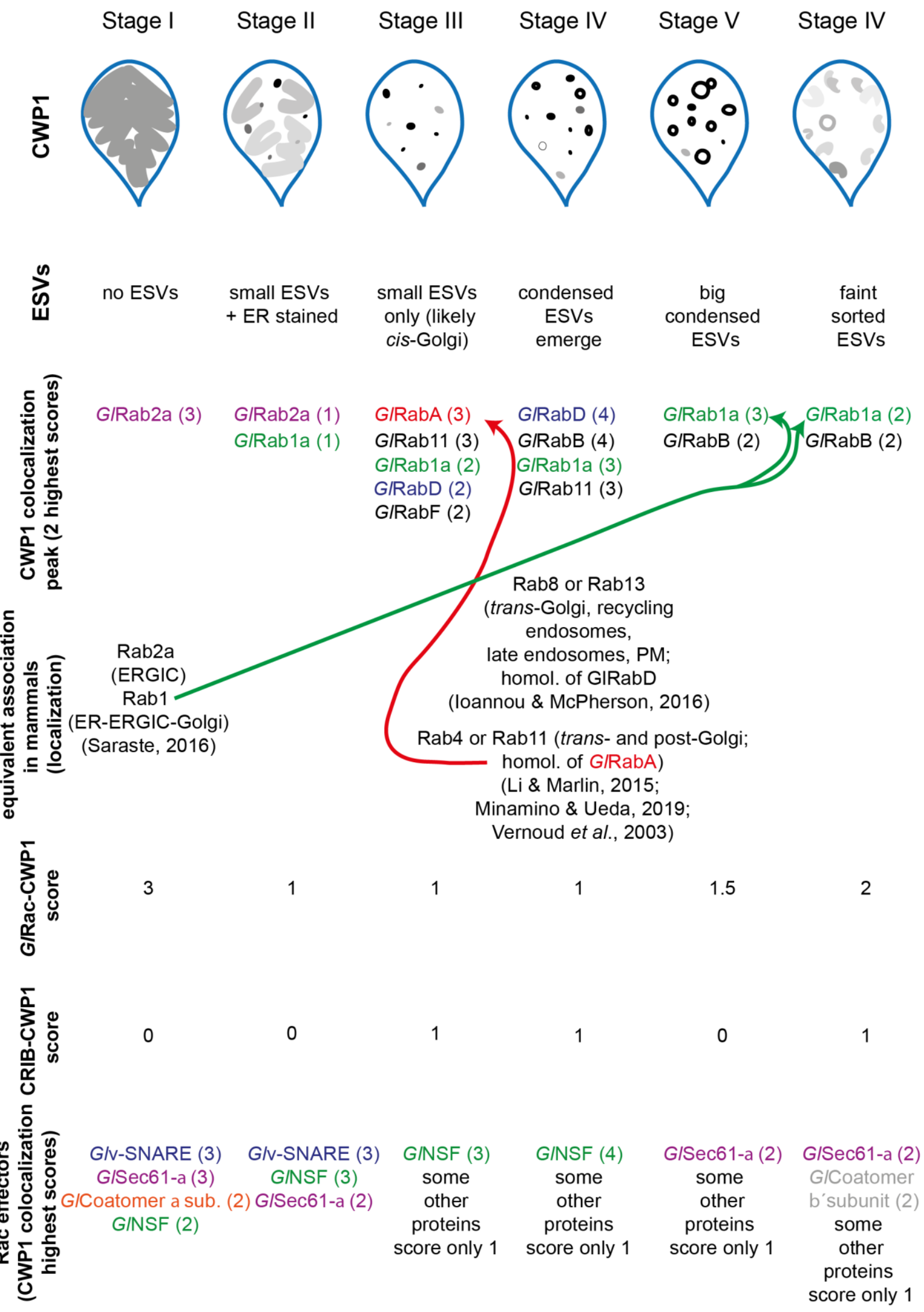

Fig. S5. Graphical summary showing the relationship of all proteins studied here relative to ESV 